\title{
A chemical study of the inner winds of asymptotic giant branch stars
}

\author{
I. Cherchneff
}

\author{
Observatoire de Genève, 51 chemin de Maillettes, 1290 Sauverny, Switzerland \\ e-mail: isabelle. cherchneff@obs . unige.ch
}

Received 9 January 2006 / Accepted 8 March 2006

\section{ABSTRACT}

\begin{abstract}
Aims. We investigate the non-equilibrium chemistry of the inner winds of AGB stars for different stages of stellar evolution, choosing a standard AGB stellar model and changing photospheric C/O ratios, to describe winds of $\mathrm{M}, \mathrm{S}$, and $\mathrm{C}$ stars. Chemical formation pathways for several important molecules and the chemistry of S stars and its implications for the nature of the dust forming in these objects are discussed.

Methods. The inner wind standard model (三gas density, temperature, and velocity) is derived from taking into account the effect of shocks induced by stellar pulsation on the gas. The chemistry consists of 68 elements and molecules and 752 chemical reactions. Molecular concentration profiles are derived by solving a system of non-linear, stiff, ordinary, coupled differential equations applied to the wind model gas parameters.

Results. We find that unexpected molecules are present in the inner winds, as a result of non-equilibrium chemistry due to shock propagation. In particular, there exists a group of molecules always formed in the inner wind of AGBs, whatever the stage of evolution of the star, i.e., $\mathrm{CO}, \mathrm{HCN}, \mathrm{CS}$, and $\mathrm{SiO}$, while other groups of species are typical of a O-rich or C-rich chemistry. The shocked regions above the photosphere, where thermal equilibrium does not apply, act as true molecular factories.
\end{abstract}

Key words. stars: AGB and post-AGB - stars: late-type - astrochemistry

\section{Introduction}

Stars on the Asymptotic Giant Branch (AGB stars) are known to develop strong molecular winds induced by dust formation and act as the main suppliers of molecules and dust to the interstellar medium. Observations at millimeter $(\mathrm{mm})$ wavelengths probe the cool, outer part of AGB winds and bring evidence of a wealth of molecular species, from simple radicals such as $\mathrm{OH}$ to more complex species like cyanopolyynes. Although many molecules are formed locally in the outer wind through various chemical and radiative processes induced by cosmic rays and the interstellar radiation field (i.e., ion-molecule reactions, photo-dissociation/ionization), some important species, named "parents", are injected to the outer envelope from deeper layers where the physical and chemical processes are different. Indeed, layers above the stellar photosphere experience the passage of stellar pulsation driven shocks which enhance the gas density and temperature, a phenomenon that triggers the formation of molecules and dust, responsible for driving the stellar mass loss through radiation pressure on grains. The existence of such regions were conjectured from theoretical models (Bertschinger \& Chevalier 1985; Bowen 1988; Fleischer et al. 1992; Willacy \& Cherchneff 1998; Woitke et al. 1999) and highlighted with the ISO SWS by Tsuji et al. (1997) and with near-infrared interferometry by Perrin et al. (2004). The "extended atmosphere" of Tsuji et al. corresponds to the "inner wind" we refer to in the present study, that is, gas layers gravitationally bound to the star and where dust can form but has not yet driven the full wind.

It is usually thought that the chemical composition of these layers results from the state of evolution of the star on the AGB, that is, the carbon-to-oxygen $(\mathrm{C} / \mathrm{O})$ ratio of the stellar photosphere. However, while it is reasonable to apply thermal equilibrium (hereafter, TE) in the photosphere because of the very large gas temperatures and densities, and then derive TE photospheric molecular abundances, this assumption does not hold for the gas layers above the photosphere through which shocks propagate. Indeed, the parameters of the post-shock gas will span wide ranges of values on very short time scales, and the chemistry will adapt to these strong variations. The first evidence of non-TE in the inner wind of AGBs was given by the observations of a few molecules which were not expected to form under TE, e.g., the ISO SWS detection of $\mathrm{CO}_{2}$ by Justtanont et al. (1996) in the O-rich AGB star NML Cygni. Theoretical models of the inner wind of AGBs which take into account the nonequilibrium chemistry induced by shock activity have been developed by Willacy \& Cherchneff (1998, hereafter WC98) for the carbon-rich star IRC+ 10216 and by Duari et al. (1999, hereafter DCW99) for the O-rich Mira IK Tau. The latter study was able to explain the formation of carbon-bearing species such as $\mathrm{HCN}$ and $\mathrm{CO}_{2}$ above the O-rich stellar photosphere. The presence of $\mathrm{HCN}$ in the inner envelope of AGBs was further confirmed by its detection at sub-millimeter wavelengths in the S star $\chi$ Cygni (Duari \& Hatchell 2000). Both theoretical studies showed that the inner winds of AGB stars experience a nonequilibrium chemistry active in levitating regions of gas whose parameters are favorable to the formation of molecules, resulting in the "extended atmosphere" observed by Tsuji et al. (1997) and Perrin et al. (2004). In particular, they showed that some molecules detected in the outer envelope of AGBs (for example, $\mathrm{HCN}$ in O-rich and $\mathrm{S}$ stars and $\mathrm{CS}$ and $\mathrm{SiO}$ in C-rich AGBs) and thought to form at large stellar radii are in fact formed in the post-shocked layers and are ejected in the outer wind as "parent" molecules.

In this paper, we study the impact of stellar evolution and photospheric $\mathrm{C} / \mathrm{O}$ ratio variations on the chemistry of the inner 
Table 1. Pre-shock, shock front, and excursion (三post-shock) gas temperature and number density as a function of position in the envelope and shock strengths. $M$ is the Mach number $\left(=v_{\mathrm{sh}} / v_{\mathrm{esc}}\right)$ associated with each shock speed.

\begin{tabular}{cccccccccc}
\hline \hline Star & Position & Shock vel. & $M$ & \multicolumn{2}{c}{ Pre-shock } & \multicolumn{2}{c}{ Shock front } & \multicolumn{2}{c}{ Start of excursion } \\
& $\left(R_{\star}\right)$ & $\left(\mathrm{km} \mathrm{s}^{-1}\right)$ & & $T_{0}(\mathrm{~K})$ & $n_{0}\left(\mathrm{~cm}^{-3}\right)$ & $T(\mathrm{~K})$ & $n\left(\mathrm{~cm}^{-3}\right)$ & $T(\mathrm{~K})$ & $n\left(\mathrm{~cm}^{-3}\right)$ \\
\hline \multirow{5}{*}{ TX Cam } & 1.0 & 25.0 & 7.41 & 2600.0 & $3.62(15)$ & 30206 & $1.99(16)$ & 6363 & $7.85(16)$ \\
& 1.5 & 20.4 & 6.84 & 2038.5 & $1.46(14)$ & 20464 & $7.91(14)$ & 4746 & $2.80(15)$ \\
& 2.0 & 17.8 & 6.45 & 1715.4 & $2.01(13)$ & 15490 & $1.07(14)$ & 3875 & $3.56(14)$ \\
& 2.5 & 15.8 & 6.17 & 1500.4 & $5.01(12)$ & 12517 & $2.65(13)$ & 3300 & $8.32(13)$ \\
& 3.0 & 14.4 & 5.95 & 1344.9 & $1.76(12)$ & 10522 & $9.25(12)$ & 2889 & $2.76(13)$ \\
\hline
\end{tabular}

envelope of AGB stars to investigate molecular formation and the chemical changes responsible for the formation of various types of dust (silicates for O-rich AGBs and amorphous carbon and silicon carbide for carbon stars). We investigate in particular C/O ratios very close to unity to discuss the chemistry of $\mathrm{S}$ stars. Finally, we wish to highlight possible observable molecular tracers for O-rich and C-rich objects in view of the new observing opportunities coming on line with the HIFI spectrometer on board of the Herschel satellite and ALMA. Indeed, the very high resolution of HIFI coupled to a frequency coverage in the Far-Infrared (FIR) and the submillimeter will allow the detection of the rotational transitions associated with the low lying vibrational states of molecules (e.g., the low energy bending modes of heavy molecules Cernicharo 1999) and the resolution of line profiles, thus providing information on the physical conditions, the dynamics, and the chemical composition of the inner winds of AGB stars. In a complementary way, ALMA will allow spatially resolved studies of nearby AGB stellar envelopes. It is therefore crucial to try and understand the chemistry acting in these inner regions from a theoretical point of view.

Section 2 gives a summary of the approach and model adopted for the study. In Sect. 3, we describe the chemistry at play. Results are fully presented and discussed in Sect. 4, in particular the presence of a group of species common to all AGB stars, molecules typical of a O-rich and C-rich chemistry, the important role of a few atomic species and radicals, and finally, the inner chemistry of S stars and its implication in dust formation. A summary and conclusions are given in Sect. 5.

\section{The inner wind model}

Layers of gas just above the photosphere experience the passage of pulsation driven shock waves, whose velocities for Mira type stars range from 20 to $30 \mathrm{~km} \mathrm{~s}^{-1}$ (Hinkle et al. 1997). The effect of these shocks on the atmosphere have been fully discussed by various authors (Fox \& Wood 1985; Bertschinger \& Chevalier 1985; Bowen 1988). We use the formalism and model developed by Cherchneff et al. (1992) and Cherchneff $(1995,1996)$, and further applied by WC98, DCW99, and Cau (2002), to describe the inner envelope of AGB stars in our study. We first define a "typical" star to apply our chemical reaction network for various C/O ratio. The elected star is TX Camelopardalis, a well-known and studied M 8.5 Mira star, with the following stellar parameters: $T_{\text {eff }}=2600 \mathrm{~K}, R_{\star}=280 R_{\odot}, P=557$ days, $M_{\star}=0.65 M_{\odot}$, and $d=317$ pc (Olofsson et al. 1991). We assume that TX Cam is pulsating in the fundamental mode. These values and assumptions are fairly standard for representing any AGB star in our study, as we do not attempt to accurately reproduce a specific object, but aim rather at understanding the chemical processes at play in the inner wind and the effect of stellar evolution on its molecular composition.

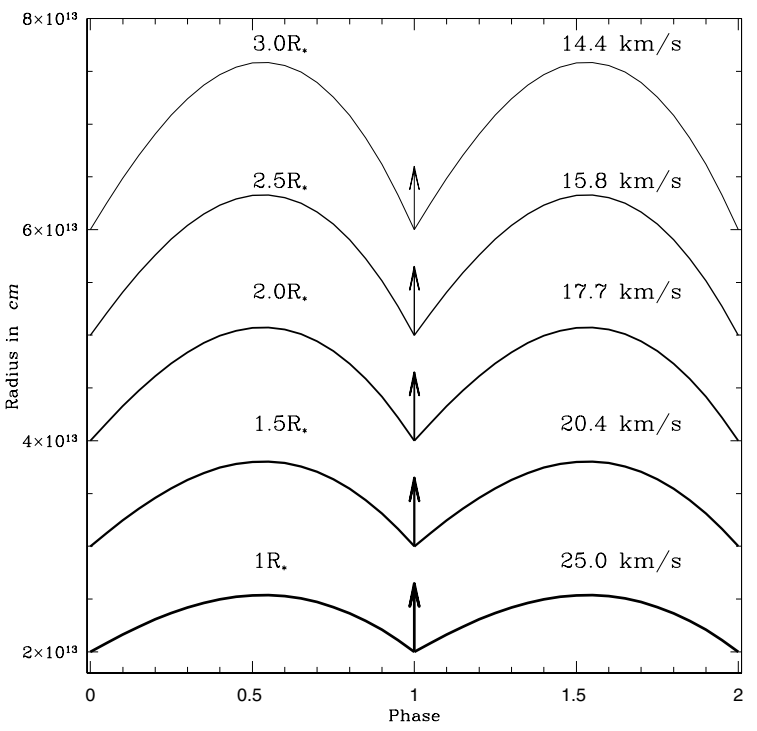

Fig. 1. Trajectories of the gas parcels induced by an initial $25 \mathrm{~km} \mathrm{~s}^{-1}$ shock as a function of position in the envelope, shock strength, and stellar pulsation phases. Arrows show the various shock positions.

In our model, the inner layers are shocked periodically and levitate above the photosphere before falling down to their initial position due to stellar gravity. We assume that the first shock steepens at $1 R_{\star}$ and further propagates outwards. We consider an initial shock velocity of $25 \mathrm{~km} \mathrm{~s}^{-1}$ at $1 R_{\star}$, a reasonable hypothesis on the ground of available measurements. This shock velocity value generates an inner envelope temperature and density profiles of the shocked, gravity-bound layers close to the photosphere (Cherchneff et al. 1992; WC98). Placing ourselves in a Lagrangian frame, we apply a non-equilibrium chemistry following the formalism of Cherchneff (1996) and WC98. Analytical parameters describing the inner layers are listed in Table 1, and Fig. 1 shows the gas trajectories above the photosphere for the initial shock velocity considered in our model of TX Cam. Each trajectory corresponds to a specific radius and shock velocity in the envelope and is made of gas excursions which repeat themselves at each stellar pulsation. An excursion represents the trajectory of the postshock gas as a function of the pulsation phase of the star.

\section{The chemistry}

The chemistry considered in this study is based on the elements presented in the stellar photosphere and all possible molecules relevant to chemistry or observations derived from them. These molecules are listed in Table 2. Elemental photospheric abundances were assumed solar except for the carbon-to-oxygen ratio which was chosen to vary from $\mathrm{C} / \mathrm{O}=0.75$ to $\mathrm{C} / \mathrm{O}=1.1$ to represent AGB stars of types $\mathrm{M}, \mathrm{S}$, and $\mathrm{C}$. The chemical 
Table 2. Chemical species considered in the present inner wind model: elements are $\mathrm{He}, \mathrm{H}, \mathrm{O}, \mathrm{C}, \mathrm{N}, \mathrm{Si}$, and $\mathrm{S}$.

\begin{tabular}{ll}
\hline \hline O-bearing & $\mathrm{O}_{2}, \mathrm{O}_{3}, \mathrm{OH}, \mathrm{H}_{2} \mathrm{O}, \mathrm{CO}, \mathrm{CO}_{2}$, \\
& $\mathrm{HCO}, \mathrm{H}_{2} \mathrm{CO}, \mathrm{SiO}, \mathrm{SiO}_{2}, \mathrm{SO}, \mathrm{SO}_{2}$, \\
& $\mathrm{SO}_{3}, \mathrm{OCS}, \mathrm{NO}, \mathrm{HNO}, \mathrm{N}_{2} \mathrm{O}, \mathrm{NO}_{2}$, \\
& $\mathrm{NO}_{3}, \mathrm{OCN}, \mathrm{HNCO}, \mathrm{HO}_{2}, \mathrm{H}_{2} \mathrm{O}_{2}$. \\
\hline C-bearing & $\mathrm{C}_{2}, \mathrm{C}_{3}, \mathrm{CH}, \mathrm{CH}_{2}, \mathrm{CH}_{3}, \mathrm{CH}_{4}$, \\
& $\mathrm{C}_{2} \mathrm{H}, \mathrm{C}_{2} \mathrm{H}_{2}, \mathrm{C}_{3} \mathrm{H}, \mathrm{C}_{3} \mathrm{H}_{2}, \mathrm{C}_{4} \mathrm{H}, \mathrm{C}_{4} \mathrm{H}_{2}$, \\
& $\mathrm{CN}, \mathrm{HCN}, \mathrm{CS}, \mathrm{HCS}, \mathrm{HCSi}, \mathrm{SiC}$, \\
& $\mathrm{SiC}_{2}, \mathrm{SiCH}_{2}$. \\
\hline Others & $\mathrm{H}_{2}, \mathrm{~S}_{2}, \mathrm{Si}_{2}, \mathrm{Si}_{3}, \mathrm{~N}_{2}, \mathrm{SiS}$, \\
& $\mathrm{HS}, \mathrm{H}_{2} \mathrm{~S}, \mathrm{SiH}, \mathrm{SiH}_{2}, \mathrm{SiH}_{3}, \mathrm{SiH}_{4}$, \\
& $\mathrm{SiN}, \mathrm{HNSi}, \mathrm{NH}, \mathrm{NH}_{2}, \mathrm{NH}_{3}, \mathrm{NS}$. \\
\hline
\end{tabular}

network includes trimolecular and bimolecular neutral-neutral processes and excludes photo-processes (dissociation and ionization) as well as ion-neutral reactions because of the weak ultraviolet stellar radiation field, the inferred absence of a chromosphere, and the very weak ionization of the post-shock gas for such shock velocities (WC98). The chemical reactions set includes 68 elements and molecules and 752 reactions. Most of the reaction rates are taken from WC98 and DWC99, with updated datas when appropriate, taken from the UMIST database RATE99 (Le Teuff et al. 2000). Unavailable reverse rates were computed following the formalism of Cherchneff et al. (1992).

We choose 5 values of $\mathrm{C} / \mathrm{O}$ ratios typical of stars ascending the AGB: for Mira-type stars, we set C/O equal to 0.75 and 0.9; for $\mathrm{S}$ stars, we investigate values of $\mathrm{C} / \mathrm{O}$ ratios equal to 0.98 (SE stars), 1, and 1.01 (SC stars); and finally, we choose $\mathrm{C} / \mathrm{O}=$ 1.1 for carbon-rich AGBs. The extreme carbon star IRC+ 10216 $(\mathrm{C} / \mathrm{O}=1,5)$ has been studied in detail by WC98.

For each value of the $\mathrm{C} / \mathrm{O}$ ratio, we run our set of non-linear, stiff, ordinary differential equations in one gas excursion and over three pulsation periods starting at $1 R_{\star}$, to check for gas parameters and helium abundance periodicity. We then move to the next closest radius, re-scale the results to the new local preshock gas density and run our system again over three pulsation periods. This approach has been followed from $1 R_{\star}$ to $5 R_{\star}$, assuming that dust condensation does not occur before $5 R_{\star}$ and using the gas parameters given in Table 1. Although using a Lagrangian formalism, we can, at the end of the calculations, derive Euler-type profiles for our species abundances which can be directly compared to observations.

\section{Results and discussion}

\subsection{Molecular content of an AGB envelope after the first shock}

We list in Table 3 a sample of species abundances at $1 R_{\star}$ resulting from the non-equilibrium chemistry induced by the propagation of the $25 \mathrm{~km} \mathrm{~s}^{-1}$ shock and present in the post-shock gas, for the two most extreme values of $\mathrm{C} / \mathrm{O}$ ratios. We also list the expected abundances from TE calculations for comparison.

Our calculations show an interesting and unexpected result: whatever the enrichment in carbon of the star, i.e., the $\mathrm{C} / \mathrm{O}$ ratio, the atomic and molecular content of the gas layers just above the stellar photosphere is very much the same, and in many cases, totally different from what would be expected from TE calculations. Indeed, we see that the abundances of the listed molecules span very similar ranges for $\mathrm{C} / \mathrm{O}=0.75$ and $\mathrm{C} / \mathrm{O}=1.1$ (see for example, $\mathrm{HCN}, \mathrm{CS}, \mathrm{CN}, \mathrm{SiS}$, and $\mathrm{SiO}$ ). Even molecules like $\mathrm{C}_{2} \mathrm{H}_{2}, \mathrm{H}_{2} \mathrm{O}, \mathrm{CO}_{2}, \mathrm{HS}, \mathrm{OH}$, and $\mathrm{SO}$ show maximum differences
Table 3. Species abundances in the post-shock gas at $1 R_{\star}$ for TE calculations and non-equilibrium chemistry.

\begin{tabular}{ccccc}
\hline \hline Species & \multicolumn{2}{c}{$\mathrm{C} / \mathrm{O}=0.75$} & \multicolumn{2}{c}{$\mathrm{C} / \mathrm{O}=1.1$} \\
& $\mathrm{TE}$ & Non TE & $\mathrm{TE}$ & Non TE \\
\hline $\mathrm{CO}$ & $5.604 \mathrm{e}-04$ & $6.037 \mathrm{e}-04$ & $7.501 \mathrm{e}-04$ & $9.587 \mathrm{e}-04$ \\
$\mathrm{HCN}$ & $1.916 \mathrm{e}-11$ & $1.610 \mathrm{e}-04$ & $1.085 \mathrm{e}-05$ & $1.633 \mathrm{e}-04$ \\
$\mathrm{CS}$ & $2.518 \mathrm{e}-11$ & $9.909 \mathrm{e}-06$ & $7.489 \mathrm{e}-06$ & $1.846 \mathrm{e}-05$ \\
$\mathrm{SiO}$ & $3.449 \mathrm{e}-05$ & $4.623 \mathrm{e}-05$ & $1.004 \mathrm{e}-08$ & $4.560 \mathrm{e}-05$ \\
$\mathrm{SiS}$ & $7.114 \mathrm{e}-09$ & $1.791 \mathrm{e}-06$ & $4.823 \mathrm{e}-07$ & $2.632 \mathrm{e}-06$ \\
$\mathrm{H}_{2} \mathrm{O}$ & $7.075 \mathrm{e}-05$ & $3.823 \mathrm{e}-04$ & $1.595 \mathrm{e}-10$ & $3.249 \mathrm{e}-05$ \\
$\mathrm{CO}_{2}$ & $2.297 \mathrm{e}-08$ & $2.815 \mathrm{e}-08$ & $6.903 \mathrm{e}-14$ & $3.663 \mathrm{e}-09$ \\
$\mathrm{C}_{2} \mathrm{H}_{2}$ & $1.285 \mathrm{e}-17$ & $9.363 \mathrm{e}-11$ & $4.565 \mathrm{e}-06$ & $2.901 \mathrm{e}-09$ \\
$\mathrm{HS}$ & $7.532 \mathrm{e}-06$ & $1.075 \mathrm{e}-05$ & $7.562 \mathrm{e}-07$ & $1.677 \mathrm{e}-06$ \\
$\mathrm{CN}$ & $3.359 \mathrm{e}-12$ & $3.296 \mathrm{e}-08$ & $1.901 \mathrm{e}-06$ & $3.569 \mathrm{e}-08$ \\
$\mathrm{OH}$ & $3.494 \mathrm{e}-05$ & $5.435 \mathrm{e}-07$ & $7.878 \mathrm{e}-11$ & $4.924 \mathrm{e}-08$ \\
$\mathrm{SO}$ & $1.820 \mathrm{e}-08$ & $4.850 \mathrm{e}-08$ & $2.147 \mathrm{e}-14$ & $7.080 \mathrm{e}-10$ \\
\hline
\end{tabular}

in abundance ratios ranging from 10 to 60 . This homogeneity of the molecular content has never been considered before and in part determines the nature of the molecules that can form at larger radii. The presence of unexpected molecules close to the photosphere for specific $\mathrm{C} / \mathrm{O}$ ratios was already discussed by WC98. In their study of the extreme carbon star IRC+ 10216 $(\mathrm{C} / \mathrm{O}=1.5)$, they showed that $\mathrm{SiO}$ formation was triggered by the presence of water $\mathrm{H}_{2} \mathrm{O}$ and the hydroxyl radical $\mathrm{OH}$ very close to the photosphere, the later reacting with atomic $\mathrm{Si}$ to form $\mathrm{SiO}$.

A first important result of this study is the confirmation that TE does not hold once shocks have propagated and that the Orich or C-rich character of the envelope of an AGB star, present in the photosphere, vanishes just above the photosphere and develops again further out in the shocked molecular layers. Indeed, we will see in Sects. 4.3 and 4.4 that this character results from the destruction and/or formation of certain molecules at a few stellar radii.

\subsection{The "common" molecules and their chemistry}

Our calculations point to the fact that at least four molecules among those listed in Table 3, whose abundances between $1 R_{\star}$ and $5 R_{\star}$ do not change drastically with $\mathrm{C} / \mathrm{O}$ ratios, exist. They are $\mathrm{CO}, \mathrm{SiO}, \mathrm{HCN}$, and $\mathrm{CS}$, and their calculated abundances with respect to the total gas number density are listed in Table 4 as a function of $\mathrm{C} / \mathrm{O}$ ratios and position in the flow. These four molecules form in high quantities whatever the $\mathrm{C} / \mathrm{O}$ ratio, a fact which implies that the chemical processes responsible for formation/destruction of these species work with the same efficiency whatever the oxygen and carbon photospheric content of the star. Apart from those four species, two molecules, $\mathrm{CN}$ and $\mathrm{HCO}$, also show abundances that stay almost constant when changing the $\mathrm{C} / \mathrm{O}$ ratio (for $\mathrm{CN}$, abundance values range from $3 \times 10^{-8}$ at $1 R_{\star}$ to $3 \times 10^{-11}$ at $5 R_{\star}$ for all $\mathrm{C} / \mathrm{O}$ ratios, while for $\mathrm{HCO}$, abundance values vary from $1.5 \times 10^{-10}$ at $1 R_{\star}$ to $1.8 \times 10^{-12}$ at $5 R_{\star}$ ). Below, we study in detail the chemistry at play for $\mathrm{CO}$, $\mathrm{SiO}, \mathrm{HCN}$, and CS and compare our results to available observational data.

\subsection{1. $\mathrm{CO}$}

Out of thermodynamical equilibrium, carbon monoxide is formed from the trimolecular reaction

$\mathrm{C}+\mathrm{O}+\mathrm{M} \rightarrow \mathrm{CO}+\mathrm{M}$ 
Table 4. Abundances between $1 R_{\star}$ to $5 R_{\star}$ for molecules common to all AGB, whatever the $\mathrm{C} / \mathrm{O}$ ratio.

\begin{tabular}{ccccccccc}
\hline \hline $\mathrm{C} / \mathrm{O}$ & $\mathrm{TE}$ & $1 R_{\star}$ & $1.5 R_{\star}$ & $2 R_{\star}$ & $2.5 R_{\star}$ & $3 R_{\star}$ & $4 R_{\star}$ & $5 R_{\star}$ \\
\hline & & \multicolumn{7}{c}{$\mathrm{CO}$} \\
\hline 0.75 & $5.604 \mathrm{e}-04$ & $6.037 \mathrm{e}-04$ & $6.114 \mathrm{e}-04$ & $6.706 \mathrm{e}-04$ & $7.096 \mathrm{e}-04$ & $7.205 \mathrm{e}-04$ & $6.678 \mathrm{e}-04$ & $6.220 \mathrm{e}-04$ \\
0.90 & $6.752 \mathrm{e}-04$ & $7.569 \mathrm{e}-04$ & $7.684 \mathrm{e}-04$ & $8.210 \mathrm{e}-04$ & $8.561 \mathrm{e}-04$ & $8.639 \mathrm{e}-04$ & $8.101 \mathrm{e}-04$ & $7.672 \mathrm{e}-04$ \\
0.98 & $7.351 \mathrm{e}-04$ & $8.362 \mathrm{e}-04$ & $8.512 \mathrm{e}-04$ & $9.031 \mathrm{e}-04$ & $9.321 \mathrm{e}-04$ & $9.328 \mathrm{e}-04$ & $8.694 \mathrm{e}-04$ & $8.314 \mathrm{e}-04$ \\
1 & $7.495 \mathrm{e}-04$ & $8.552 \mathrm{e}-04$ & $8.725 \mathrm{e}-04$ & $9.245 \mathrm{e}-04$ & $9.579 \mathrm{e}-04$ & $9.620 \mathrm{e}-04$ & $8.831 \mathrm{e}-04$ & $8.408 \mathrm{e}-04$ \\
1.01 & $7.501 \mathrm{e}-04$ & $8.719 \mathrm{e}-04$ & $8.964 \mathrm{e}-04$ & $9.975 \mathrm{e}-04$ & $9.925 \mathrm{e}-04$ & $9.772 \mathrm{e}-04$ & $9.006 \mathrm{e}-04$ & $8.503 \mathrm{e}-04$ \\
1.1 & $7.501 \mathrm{e}-04$ & $9.587 \mathrm{e}-04$ & $1.006 \mathrm{e}-03$ & $1.008 \mathrm{e}-03$ & $1.009 \mathrm{e}-03$ & $9.915 \mathrm{e}-04$ & $9.099 \mathrm{e}-04$ & $8.577 \mathrm{e}-04$ \\
\hline & & & & & \\
0.75 & $3.449 \mathrm{e}-05$ & $4.623 \mathrm{e}-05$ & $4.569 \mathrm{e}-05$ & $4.804 \mathrm{e}-05$ & $4.801 \mathrm{e}-05$ & $4.737 \mathrm{e}-05$ & $4.420 \mathrm{e}-05$ & $4.248 \mathrm{e}-05$ \\
0.90 & $3.390 \mathrm{e}-05$ & $4.604 \mathrm{e}-05$ & $4.613 \mathrm{e}-05$ & $4.813 \mathrm{e}-05$ & $4.800 \mathrm{e}-05$ & $4.721 \mathrm{e}-05$ & $4.332 \mathrm{e}-05$ & $4.148 \mathrm{e}-05$ \\
0.98 & $1.420 \mathrm{e}-05$ & $4.570 \mathrm{e}-05$ & $4.616 \mathrm{e}-05$ & $4.791 \mathrm{e}-05$ & $4.769 \mathrm{e}-05$ & $4.657 \mathrm{e}-05$ & $4.131 \mathrm{e}-05$ & $3.962 \mathrm{e}-05$ \\
1 & $6.396 \mathrm{e}-07$ & $4.551 \mathrm{e}-05$ & $4.617 \mathrm{e}-05$ & $4.783 \mathrm{e}-05$ & $4.728 \mathrm{e}-05$ & $4.300 \mathrm{e}-05$ & $3.069 \mathrm{e}-05$ & $3.309 \mathrm{e}-05$ \\
1.01 & $6.232 \mathrm{e}-08$ & $4.623 \mathrm{e}-05$ & $4.682 \mathrm{e}-05$ & $4.602 \mathrm{e}-05$ & $3.895 \mathrm{e}-05$ & $2.774 \mathrm{e}-05$ & $1.357 \mathrm{e}-05$ & $2.239 \mathrm{e}-05$ \\
1.1 & $1.004 \mathrm{e}-08$ & $4.560 \mathrm{e}-05$ & $4.014 \mathrm{e}-05$ & $3.788 \mathrm{e}-05$ & $2.389 \mathrm{e}-05$ & $1.123 \mathrm{e}-05$ & $6.901 \mathrm{e}-06$ & $8.496 \mathrm{e}-06$ \\
\hline & & & & & \\
0.75 & $1.916 \mathrm{e}-11$ & $1.610 \mathrm{e}-04$ & $1.540 \mathrm{e}-04$ & $9.063 \mathrm{e}-05$ & $5.120 \mathrm{e}-05$ & $2.732 \mathrm{e}-05$ & $7.574 \mathrm{e}-06$ & $1.136 \mathrm{e}-05$ \\
0.90 & $8.589 \mathrm{e}-11$ & $1.632 \mathrm{e}-04$ & $1.527 \mathrm{e}-04$ & $9.474 \mathrm{e}-05$ & $5.541 \mathrm{e}-05$ & $3.107 \mathrm{e}-05$ & $8.983 \mathrm{e}-06$ & $1.166 \mathrm{e}-05$ \\
0.98 & $4.645 \mathrm{e}-09$ & $1.633 \mathrm{e}-04$ & $1.498 \mathrm{e}-04$ & $9.235 \mathrm{e}-05$ & $5.518 \mathrm{e}-05$ & $3.064 \mathrm{e}-05$ & $7.811 \mathrm{e}-06$ & $9.773 \mathrm{e}-06$ \\
1 & $1.739 \mathrm{e}-07$ & $1.644 \mathrm{e}-04$ & $1486 \mathrm{e}-04$ & $9.126 \mathrm{e}-05$ & $4.732 \mathrm{e}-05$ & $1.370 \mathrm{e}-05$ & $4.770 \mathrm{e}-06$ & $6.448 \mathrm{e}-06$ \\
1.01 & $1.807 \mathrm{e}-06$ & $1.630 \mathrm{e}-04$ & $1.420 \mathrm{e}-04$ & $3.406 \mathrm{e}-05$ & $1.610 \mathrm{e}-05$ & $9.210 \mathrm{e}-06$ & $3.530 \mathrm{e}-06$ & $5.360 \mathrm{e}-06$ \\
1.1 & $1.085 \mathrm{e}-05$ & $1.633 \mathrm{e}-04$ & $6.184 \mathrm{e}-05$ & $2.877 \mathrm{e}-05$ & $1.583 \mathrm{e}-05$ & $9.626 \mathrm{e}-06$ & $4.503 \mathrm{e}-06$ & $5.814 \mathrm{e}-06$ \\
\hline
\end{tabular}

and the bimolecular process

$\mathrm{C}+\mathrm{OH} \rightarrow \mathrm{CO}+\mathrm{H}$

The former reaction represents about $64 \%$ of the formation and the latter, about $24 \%$, independent of the $\mathrm{C} / \mathrm{O}$ ratio. Inspection of Table 3 reveals that at $1 R_{\star}$, the hydroxyl radical $\mathrm{OH}$ is present in $\mathrm{M}, \mathrm{S}$, and $\mathrm{C}$ stars as a result of shock chemistry (see Sect. 4.3.1) and can therefore induce Reaction 2 for stars with $\mathrm{C} / \mathrm{O} \geq 1$. The rate of Reaction 1 has an activation energy barrier and will then be efficient at high gas densities and temperatures, whereas Reaction 2 has a fast rate free of the activation energy barrier and thus ensures $\mathrm{CO}$ formation at any position in the wind. The destruction of CO occurs via the formation process of CS (87-90\% of the destruction processes)

$\mathrm{CO}+\mathrm{S} \rightarrow \mathrm{CS}+\mathrm{O}$

and the formation of $\mathrm{HCO}$ (9-12\% of the destruction processes)

$\mathrm{CO}+\mathrm{H}_{2} \rightarrow \mathrm{HCO}+\mathrm{H}$

We see from Table 4 that the $\mathrm{CO}$ abundances increase with increasing $\mathrm{C} / \mathrm{O}$ ratio and decrease with larger radius values. Although the formation and destruction rates are higher in oxygen stars than in their carbon counterparts, the net formation rate is slightly larger in the case of carbon stars, leading to larger $\mathrm{CO}$ abundances for larger $\mathrm{C} / \mathrm{O}$ ratios. The abundance decreases with radius, whatever the $\mathrm{C} / \mathrm{O}$ ratio, as a result of a decrease in the trimolecular Reaction 1 efficiency at lower gas densities. We also notice that non-equilibrium $\mathrm{CO}$ abundances are always greater than TE values by $\approx 11 \%$ for $M$ stars to $14 \%$ for carbon stars. For TX cam, the CO abundance at $5 R_{\star}$ is twice the value assumed by Olofsson et al. (1991) and Knapp \& Morris (1985) for O-rich AGBs. This would result in a mass loss increase for this star of $\approx 1.86$, using the mass loss equation derived by Knapp \& Morris. For carbon stars, on the other hand, our CO model abundance is consistent with the value assumed by Knapp \& Morris and coherent with the model values derived for IRC $+10216\left(9.9 \times 10^{-4}\right)$ by WC98.

\subsection{2. $\mathrm{SiO}$}

As already stressed by WC98 in their study of IRC+ 10216, the chemistry of $\mathrm{SiO}$ is linked to the presence of the hydroxyl radical $\mathrm{OH}$, which is formed from shock chemistry in the inner wind. We confirm this chemical formation route for $\mathrm{SiO}$ for the various $\mathrm{C} / \mathrm{O}$ ratios of our study. Indeed, the reaction

$\mathrm{Si}+\mathrm{OH} \rightarrow \mathrm{SiO}+\mathrm{H}$

is responsible for $84 \%$ of $\mathrm{SiO}$ formation at $1 R_{\star}$ and at the beginning of each excursion of gas, the equivalent of the fast chemistry zone defined by WC98. The main destruction channel at this location is the opposite of Reaction 5. OH further alters the $\mathrm{SiO}$ abundance at larger radii by the formation of $\mathrm{SiO}_{2}$ via the reaction

$\mathrm{SiO}+\mathrm{OH} \rightarrow \mathrm{SiO}_{2}+\mathrm{H}$

For $\mathrm{C} / \mathrm{O}<1$, Reactions 5 and 6 and their reverse processes are active from 1 to $5 R_{\star}$ because of the sustained formation of $\mathrm{OH}$, enabling the formation of $\mathrm{SiO}$ and its very efficient conversion into $\mathrm{SiO}_{2}$ at these radii. For $\mathrm{C} / \mathrm{O}>1, \mathrm{OH}$ is formed at $1 R_{\star}$ but its formation is not maintained constantly within the inner wind because of the gradual absence of oxygen atoms (see Sect. 4.3.1). Therefore, the amount of $\mathrm{SiO}$ is maximum close to the star, and gradually decreases, while $\mathrm{SiO}_{2}$ becomes negligible at radii greater than $1 R_{\star}$. The lack of $\mathrm{OH}$ in the inner wind of 
carbon stars is thus responsible for their inability to form silicate dust despite their large $\mathrm{SiO}$ content.

Our derived, almost constant, high $\mathrm{SiO}$ abundances (about $\left.4 \times 10^{-5}\right)$ before condensation of dust for TX Cam $(\mathrm{C} / \mathrm{O}=0.75)$ are in good agreement with the abundances derived from thermal mm line emission observations of Miras by González Delgado et al. (2003) and Schöier et al. (2004). For carbon stars, we see that the present $\mathrm{SiO}$ abundances decrease with increasing $\mathrm{C} / \mathrm{O}$ ratios and radius, with a minimum value at the dust formation radius of about $8 \times 10^{-6}$. For IRC $+10216(\mathrm{C} / \mathrm{O}=1.5)$, WC98 found an abundance at $5 R_{\star}$ of $2 \times 10^{-7}$, confirming these trends. These values are in good agreement with the mm survey of several carbon stars, including IRC +10216 , carried by Woods et al. (2003). From the chemistry, we should therefore expect higher $\mathrm{SiO}$ abundances in the inner wind of O-rich stars than in their carbon counterparts, a fact that was already observed by Bujarrabal et al. (1994).

\subsection{3. $\mathrm{HCN}$}

$\mathrm{HCN}$ is another example of the importance of the chemistry of shocks just above the photosphere. For all the $\mathrm{C} / \mathrm{O}$ ratios of this study, the formation mechanism of $\mathrm{HCN}$ is directly linked to its radical $\mathrm{CN}$ by

$\mathrm{CN}+\mathrm{H}_{2} \rightarrow \mathrm{HCN}+\mathrm{H}$,

where the destruction channel is the reverse reaction, that is, the attack of HCN by atomic hydrogen. These two processes take place from $1 R_{\star}$ to $5 R_{\star}$, at any phase of the gas excursions. If we look at the chemical reactions responsible for formation and destruction of $\mathrm{CN}$ in these regions, they are respectively the opposite of Reaction 7 and Reaction 7 itself. Therefore, to understand the presence of $\mathrm{HCN}$ in large quantities for any $\mathrm{C} / \mathrm{O}$ ratios, we have to investigate the chemistry in the "very fast chemistry" zone described by WC98, that is the narrow region after the shock front itself. Doing so at $1 R_{\star}$, we see that CN is forming after the shock front by the following routes

$\mathrm{N}+\mathrm{CH} \rightarrow \mathrm{CN}+\mathrm{H}$

and

$\mathrm{N}+\mathrm{CS} \rightarrow \mathrm{CN}+\mathrm{S}$.

Reaction 8, with its fast rate, is the dominant formation route for $\mathrm{CN}$, accounting for 55 to $72 \%$ of the formation rate. As for the radical $\mathrm{CH}$, its formation occurs simultaneously to Reaction 8 in the shock front by

$\mathrm{H}+\mathrm{C}_{2} \rightarrow \mathrm{CH}+\mathrm{C}$.

The rate for Reaction 10 has a very high activation barrier of $30450 \mathrm{~K}$ (or $4.2 \times 10^{-12} \mathrm{erg}$ ) that inhibits fast $\mathrm{CH}$ formation out of the shock front. Shocks therefore trigger the $\mathrm{CN}$ and further $\mathrm{HCN}$ formation in the gas. Again, the formation processes of these molecules do not depend on the photospheric $\mathrm{C} / \mathrm{O}$ ratios, but rather on the physical parameters of the shocked gas, and the aftermath is the formation of $\mathrm{HCN}$ in large quantities in both O-rich, $\mathrm{S}$, and $\mathrm{C}$-rich stars above the photosphere. Once $\mathrm{HCN}$ formation starts close to the star, the destruction/formation processes at larger radii involve essentially Reaction 7 and its reverse process, a self-feeding mechanism between the molecule and its radical, resulting in HCN survival whatever the chemistry at play locally.

For IRC +10216 , several vibrational transitions of $\mathrm{HCN}$ have been observed with the ISO SWS in absorption between 2.5 and $3.6 \mu \mathrm{m}$ and in emission around $14 \mu \mathrm{m}$ by Cernicharo et al. (1999), who derived a HCN abundance of $\approx 1 \times 10^{-5}$, in good agreement with the calculated value of WC98 $\left(\approx 3 \times 10^{-5}\right.$ at $4 R_{\star}$ and before dust condensation). Aoki et al. (1998, Paper II, 1999) also observed the same HCN transitions in several carbon stars (of type $\mathrm{SC}(\mathrm{C} / \mathrm{O}=1.01)$ and $\mathrm{N}(\mathrm{C} / \mathrm{O}=1.1)$ ) along with $\mathrm{CH}$ vibration-rotations transitions between 3.3 and $3.8 \mu \mathrm{m}$ with the ISO SWS. Therefore, the simultaneous detection of HCN and $\mathrm{CH}$ in the flow supports the validity of our chemical channels for $\mathrm{HCN}$ formation.

For O-rich stars and S stars, $\mathrm{HCN}$ has long been detected at $\mathrm{mm}$ wavelengths. The HCN abundance observed by Olofsson et al. (1991) in TX Cam and by Lindqvist et al. (1988) in several other O-rich Miras could not be reconciled with theoretical models of the outer envelopes, including photo-dissociation processes. They argued that the observed $\mathrm{CN} / \mathrm{HCN}$ ratio in these stars implied an inner wind origin. The more recent $\mathrm{mm}$ survey of Bieging et al. (2000), including 30 AGB stars of spectral type $\mathrm{M}, \mathrm{S}$, and $\mathrm{C}$, confirms the presence of $\mathrm{HCN}$ in O-rich Miras and in $\mathrm{S}$ stars and the need of an internal origin for $\mathrm{HCN}$ to explain the observed HCN line intensity ratios. In particular, they found that $\mathrm{M}$ and $\mathrm{S}$ stars present a strong correlation between $\mathrm{HCN} / \mathrm{SiO}$ integrated line intensity ratios and mass loss, pointing to the fact that HCN must form near the stellar photosphere for these stars. Furthermore, their integrated line intensity ratio for $\mathrm{HCN}$ and $\mathrm{SiO}$ show a trend of increasing values from $\mathrm{M}$ to $\mathrm{C}$ stars, with almost similar values for $\mathrm{S}$ and $\mathrm{M}$ stars. Our present results show the same trend if one considers $\mathrm{HCN}$ to $\mathrm{SiO}$ abundance ratios at $5 R_{\star}$ as a function of $\mathrm{C} / \mathrm{O}$ ratios, with closer values between $\mathrm{M}$ and $\mathrm{S}$ stars. Hence, we confirm that $\mathrm{HCN}$ is present in both $\mathrm{M}, \mathrm{S}$, and $\mathrm{C}$ stars, with maximum abundances for $\mathrm{S}$ stars, and that it is formed in the inner winds of these objects. Finally, high excitation ro-vibrational transitions of $\mathrm{HCN}$ were also detected in the S star $\chi$ Cygni $(C / O=0.95)$ (Duari \& Hatchell 2000), implying the presence of $\mathrm{HCN}$ at radii $<20 R_{\star}$ to satisfy excitation requirements.

\subsubsection{CS}

Inspection of Table 4 shows that CS forms in quantities comparable to those of $\mathrm{HCN}$ and should be present with high abundance values in all AGB stars. For all C/O ratios, the dominant formation pathway to CS close to the star occurs in the fast chemistry zone of the gas parcel excursion and corresponds to

$\mathrm{C}+\mathrm{SO} \rightarrow \mathrm{CS}+\mathrm{O}$

followed by

$\mathrm{S}+\mathrm{CN} \rightarrow \mathrm{CS}+\mathrm{N}$

at larger radii and/or towards the end of the excursion. The main destruction channel is not one of the reverse processes of the above two reactions, but the attack by atomic oxygen

$\mathrm{CS}+\mathrm{O} \rightarrow \mathrm{CO}+\mathrm{S}$

efficient again close to the star. A second process contributes to destruction for $\mathrm{C} / \mathrm{O}>1$, and involve $\mathrm{HS}$ formation

$\mathrm{H}+\mathrm{CS} \rightarrow \mathrm{HS}+\mathrm{C}$.

From Table 4, we see that the CS abundances show a maximum value at $2 R_{\star}$ for $\mathrm{C} / \mathrm{O} \leq 1.01$, followed by a decrease at larger radii. For $M$ stars, the decrease is continuous until the dust formation radius is reached, while it stabilizes for $\mathrm{C} / \mathrm{O}=0.98,1$, 


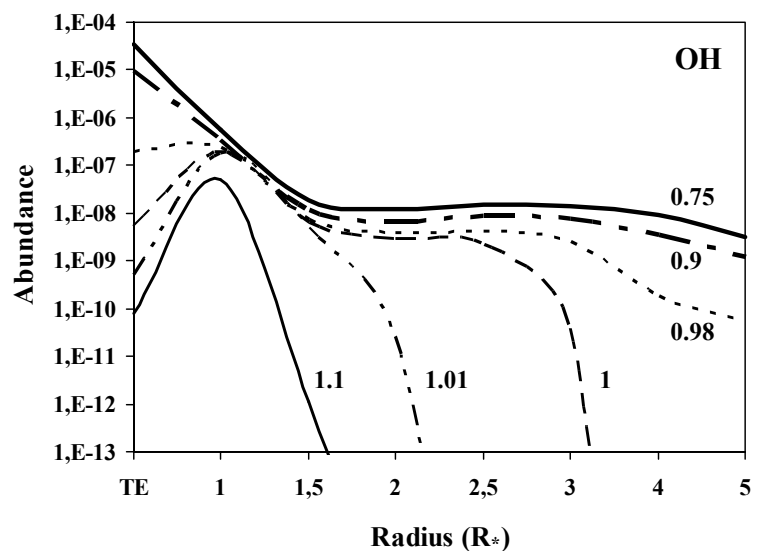

Fig. 2. $\mathrm{OH}$ abundances versus $\mathrm{C} / \mathrm{O}$ ratio (curve labels) and stellar radius. TE abundances are also plotted on the left-hand side of the radius axis for comparison.

and 1.01. Abundances in C-rich stars show a different trend, with a constant decrease up to $3 R_{\star}$ and stabilization afterwards. The $\mathrm{S}$ stars show the highest CS abundances over the range of radii because $\mathrm{CN}$ has higher net formation rates in these stars due to the large $\mathrm{HCN}$ abundances.

On the observational front, infrared fundamental and firstovertone bands were detected by Aoki et al. (1998, Paper II) with the ISO SWS in the SC star WZ Cas and the N star TX Psc. At millimeter wavelengths, Olofsson et al. (1998) studied a large sample of oxygen and carbon stars and reported detection of CS lines in all carbon objects and in a few oxygen-rich stars. They derived CS to CO line intensity ratios that are much higher for $\mathrm{C}$ stars than for $\mathrm{M}$ objects by a factor of 8 . Although no direct comparisons can be made as our calculations use a unique stellar model, our results support this trend for radii $>3 R_{\star}$, with CS to $\mathrm{CO}$ abundance ratios higher by a factor of 4 in carbon stars than in O-rich Miras. Furthermore, in their theoretical model of the outer chemistry of the carbon star IRC +10216 , Millar et al. (2001) argue for the need of CS injection from the inner envelope to match CS abundances derived by $\mathrm{mm}$ line observations.

\subsection{Molecules typical of oxygen chemistry}

From the results above, we see that some molecules are common to the inner gas layers of any AGB stars, and should further travel through the intermediate and outer envelopes untouched, except for $\mathrm{SiO}$, whose abundance is expected to be depleted because of its role in the nucleation of dust and its further freezing on grain surfaces in the intermediate wind of O-rich stars. On the other hand, some other molecules are only present in the inner winds of stars of specific stellar type. In this section, we investigate the formation processes of those species for oxygen-rich objects.

\subsection{1. $\mathrm{OH}$ and $\mathrm{H}_{2} \mathrm{O}$}

The present calculations show that the hydroxyl radical $\mathrm{OH}$ plays an important role in the inner wind of AGBs as a trigger to molecular formation. Indeed, we saw in Sect. 4.2 that $\mathrm{OH}$ is responsible for the formation of $\mathrm{CO}$ and $\mathrm{SiO}$ in both O-rich and carbon inner envelopes. We will see below that it is also responsible for $\mathrm{CO}_{2}$ formation in O-rich stars. The abundances of $\mathrm{OH}$ as a function of $\mathrm{C} / \mathrm{O}$ ratio and stellar radius are shown in Fig. 2. At $1 R_{\star}, \mathrm{OH}$ is present in any AGB with abundances extremely different from those predicted by TE equilibrium calculations

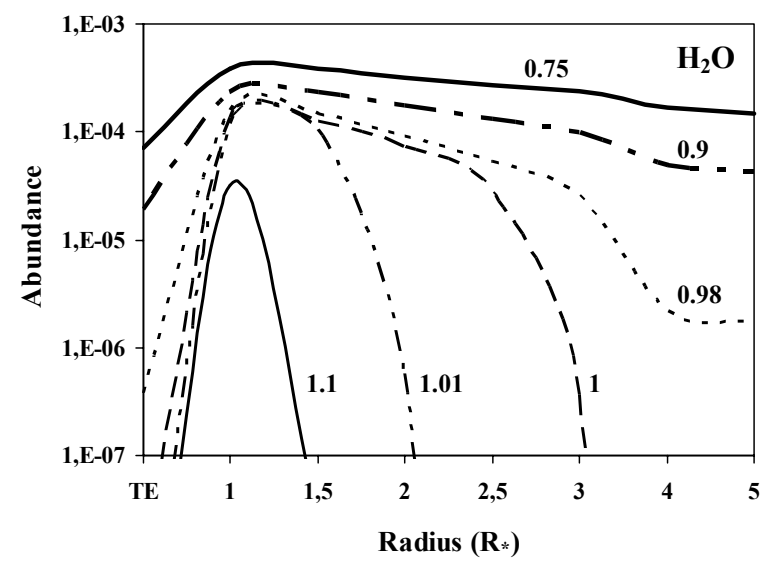

Fig. 3. $\mathrm{H}_{2} \mathrm{O}$ abundances versus $\mathrm{C} / \mathrm{O}$ ratio (curve labels) and stellar radius. TE abundances are also plotted on the left-hand side of the radius axis for comparison.

and forms at small phases of the gas excursion (shortly after the passage of the shock) by the reaction

$\mathrm{O}+\mathrm{H}_{2} \rightarrow \mathrm{OH}+\mathrm{H}$

with a $91 \%$ efficiency and is destroyed by the opposite chemical process.

Water forms at any phase of the pulsation and any radius in the flow from reaction

$\mathrm{OH}+\mathrm{H}_{2} \rightarrow \mathrm{H}_{2} \mathrm{O}+\mathrm{H}$

which is efficient at $100 \%$. The main destruction process is the reverse of Reaction 16. Reaction rates for both Reactions 15 and 16 have a high temperature dependence and an activation barrier which imply that they are fast enough for high temperatures ranges $\left(k_{15,16}=1 \times 10^{-11} \mathrm{~cm}^{3} \mathrm{~s}^{-1}\right.$ at $\left.2000 \mathrm{~K}\right)$, but drop quickly for temperatures $<1500 \mathrm{~K}$. This results in a maximum net formation rate for $\mathrm{OH}$ and $\mathrm{H}_{2} \mathrm{O}$ in the gas layers close to the photosphere through which a shock front has just passed.

$\mathrm{H}_{2} \mathrm{O}$ abundances are shown in Fig. 3. In the case of TX Cam $(\mathrm{C} / \mathrm{O}=0.75)$, the calculated abundance is $\approx 1 \times 10^{-4}$ in the inner wind. From Reaction 16, we see that $\mathrm{H}_{2} \mathrm{O}$ abundances are highly dependent on $\mathrm{OH}$ and its formation efficiency. At any radius and for any $\mathrm{C} / \mathrm{O}$ ratio, the net rate for $\mathrm{H}_{2} \mathrm{O}$ formation is always positive while that of $\mathrm{OH}$ is negative, implying a constant conversion of $\mathrm{OH}$ into $\mathrm{H}_{2} \mathrm{O}$ in the wind. For $\mathrm{C} / \mathrm{O}<1$, the $\mathrm{OH}$ abundance at $1 R_{\star}$ is larger by a factor of 10 than for $\mathrm{C} / \mathrm{O}>1$, while $\mathrm{H}_{2}$ abundances are comparable, resulting in a higher formation rate for water in O-rich AGBs. In C-stars, the gradual depletion of atomic oxygen in the flow results in very low $\mathrm{OH}$ abundances for $R>1 R_{\star}$ and a failure to sustain $\mathrm{H}_{2} \mathrm{O}$ formation (see Fig. 3).

Observation of $\mathrm{H}_{2} \mathrm{O}$ IR transitions in O-rich AGBs with the ISO SWS/LWS was reported by various authors (Neufeld et al. 1996; Tsuji et al. 1997; Truong-Bach et al. 1999; Yamamura et al. 1999; Markwick \& Millar 2000; Tsuji 2000; Ryde \& Eriksson 2002), and our present model values are in good agreement with abundances derived from ISO data (Truong-Bach et al.) and from modeling of the $183 \mathrm{GHz}$ water maser emission line by González-Alfonso \& Cernicharo (1999).

\subsection{2. $\mathrm{CO}_{2}, \mathrm{SO} / \mathrm{SO}_{2}$ and $\mathrm{HS}$}

Detection of the $4.2 \mu \mathrm{m}$ absorption band of carbon dioxide $\mathrm{CO}_{2}$ in O-rich AGBs with the ISO SWS was first reported by Justtanont et al. (1996), followed by Tsuji et al. (1997), 


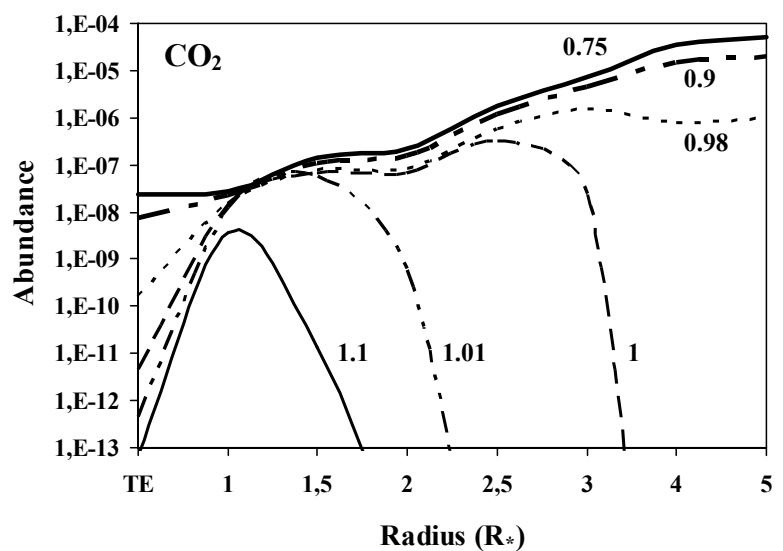

Fig. 4. $\mathrm{CO}_{2}$ abundances versus $\mathrm{C} / \mathrm{O}$ ratio (curve labels) and stellar radius. TE abundances are also plotted on the left-hand side of the radius axis for comparison.

Cami et al. (2000), and Justtanont et al. (2004). It was the first time that $\mathrm{CO}_{2}$ was detected in Miras stars and this detection was surprising at that time as it was thought, on the basis of TE considerations, that carbon was mostly locked up in $\mathrm{CO}$ and could not be available for the formation of other carbon-bearing molecules. DCW99 modeled the chemistry active in the inner wind of the O-rich AGB IK Tau and reported successful $\mathrm{CO}_{2}$ formation in the shocked gas via the reaction first proposed by Nercessian et al. (1989) for the outer envelope:

$\mathrm{OH}+\mathrm{CO} \rightarrow \mathrm{CO}_{2}+\mathrm{H}$.

Our present model $\mathrm{CO}_{2}$ abundances are illustrated in Fig. 4 for the various $\mathrm{C} / \mathrm{O}$ ratios studied. For the particular case of TX Cam, we obtained an abundance of $5 \times 10^{-5}$ at $5 R_{\star}$, coherent with that derived by DCW99 for IK Tau. Reaction 17 contributes to $94 \%$ of $\mathrm{CO}_{2}$ formation at the beginning of the gas excursion for any radius, as its chemical rate is fairly independent of temperature. The following additional formation channel contributes to formation to a $50 \%$ level at later phases of the excursions

$\mathrm{H}_{2} \mathrm{O}+\mathrm{CO} \rightarrow \mathrm{CO}_{2}+\mathrm{H}_{2}$,

while the destruction channels are the opposite of Reactions 17 and 18 . The net formation rate for $\mathrm{CO}_{2}$ is always positive, whatever the position in the flow, resulting in the regular abundance increase illustrated in Fig. 4. The final abundances for $\mathrm{C} / \mathrm{O}<1$ are far superior to those under thermal equilibrium and definitely high for stars with $\mathrm{C} / \mathrm{O}<0.9$. The above chemical processes are also active in carbon stars, although $\mathrm{CO}_{2}$ stops forming at radii $>1 R_{\star}$ because of the lack of $\mathrm{OH}$ at these radii in stars with $\mathrm{C} / \mathrm{O}>1 . \mathrm{CO}_{2}$ is then the result of shock chemistry above the stellar photosphere and a good candidate for discriminating between O-rich and C-rich AGB stars.

SO has long been detected in the outer envelope of O-rich Miras at mm wavelengths (Omont et al. 1993; Bujarrabal et al. 1994). Willacy \& Millar (1997) modeled the chemistry of the outer envelopes of several O-rich AGBs, including TX Cam, for which they found a SO abundance lower than that observed by Bujarrabal et al. They assumed no SO injection from the inner wind at large radii but in-situ formation processes only. Our model abundances of SO are illustrated in Fig. 5, and our model value for TX Cam at $5 R_{\star}$ is $3.4 \times 10^{-7}$. Assuming that $\mathrm{SO}$ is injected to large radii in the envelope, this value added to that derived from local formation processes could boost the outer SO

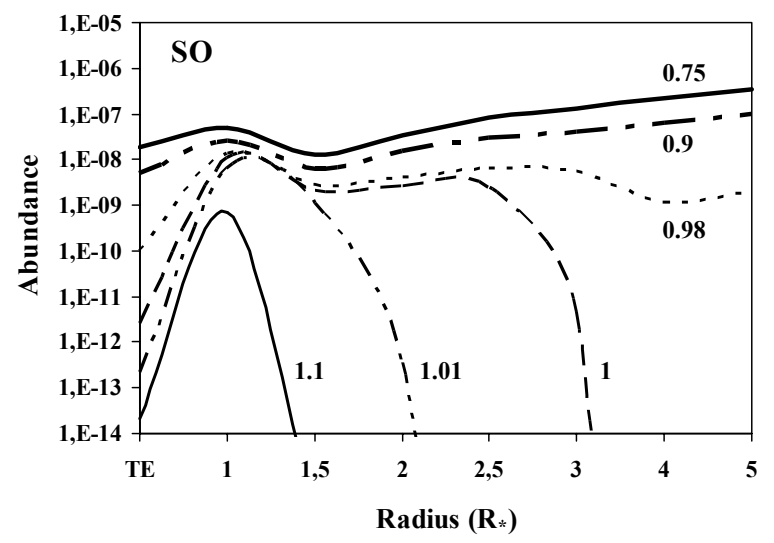

Fig. 5. SO abundances versus $\mathrm{C} / \mathrm{O}$ ratio (curve labels) and stellar radius. TE abundances are also plotted on the left-hand side of the radius axis for comparison.

abundance to better match the observed value of $1.8 \times 10^{-6} \mathrm{de}-$ rived by Bujarrabal et al. In our model, $\mathrm{SO}$ is formed at high gas density and temperature from the trimolecular reaction

$\mathrm{S}+\mathrm{O}+\mathrm{M} \rightarrow \mathrm{SO}+\mathrm{M}$

which accounts for $73 \%$ of the formation, and by the bimolecular channel

$\mathrm{S}+\mathrm{CO} \rightarrow \mathrm{SO}+\mathrm{C}$

which provides for the $27 \%$ left. Towards lower gas temperatures, the dominant formation process is

$\mathrm{S}+\mathrm{OH} \rightarrow \mathrm{SO}+\mathrm{H}$.

The dominant destruction process for any position in the flow is attack by atomic hydrogen via

$\mathrm{SO}+\mathrm{H} \rightarrow \mathrm{OH}+\mathrm{S}$.

All the above reaction channels take place in both O-rich and C-rich AGBs. At small stellar radii, the SO net formation rate is positive, with slightly lower values for carbon stars than for M Miras. On the other hand, Reaction 21 is highly prohibited at larger radii in carbons stars due to the lack of $\mathrm{OH}$ radicals, resulting in a sharp drop in the net formation rate and the SO abundances of Fig. 5. Therefore, SO is typical of O-rich Miras envelopes, as already observed by Bujarrabal et al. (1994) who derived much lower SO abundances in C-rich objects, if any, than in O-rich AGBs.

As for $\mathrm{SO}_{2}$, the main formation channel in this study is

$\mathrm{SO}+\mathrm{OH} \rightarrow \mathrm{SO}_{2}+\mathrm{H}$

while its destruction occurs via the reverse reaction. For $\mathrm{C} / \mathrm{O}=$ 0.75 , our model $\mathrm{SO}_{2}$ abundance at $1 R_{\star}$ is $1.3 \times 10^{-12}$ and decreases at larger radii by a factor 10 . The abundances get even smaller with increasing values of $\mathrm{C} / \mathrm{O}$ ratios, indicating that our model cannot form large quantities of $\mathrm{SO}_{2}$ in any AGB star.

The detection of $\mathrm{SO}_{2}$ with the ISO SWS in several O-rich AGBs by Yamamura et al. (1999) implies a formation site close to the star and an abundance spanning the range $10^{-8}-10^{-7}$, slightly below $\mathrm{SO}$ values. Hence, our model fails in reproducing observations, partly due to the limited number of reactions involved in the $\mathrm{SO}_{2}$ formation scheme and the lack of measured reaction rates. Other formation processes must then be advocated. The absence of a chromosphere and the assumption of neutral 


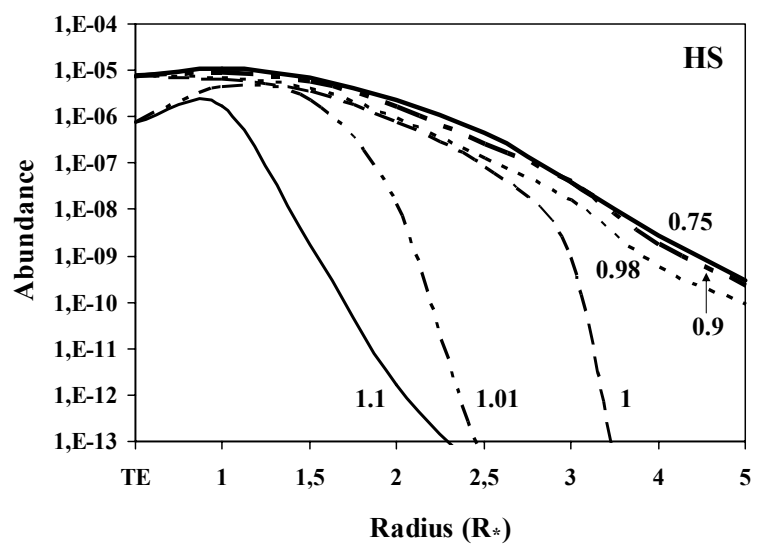

Fig. 6. HS abundances versus $\mathrm{C} / \mathrm{O}$ ratio (curve labels) and stellar radius. $\mathrm{TE}$ abundances are also plotted on the left-hand side of the radius axis for comparison.

shocks in our model excludes ion-neutral reactions and photoprocesses, in particular photo-dissociation, which could play a role in the chemistry of some species such as $\mathrm{SO}_{2}$.

Finally, we present our results for HS in Fig. 6. We can see that HS is present in all stars at $1 R_{\star}$, but that its abundance decreases with radius, slowly in O-rich and $\mathrm{S}$ stars, but very rapidly, to become negligible at $2 R_{\star}$, for carbon stars. Very much like the $\mathrm{CN} / \mathrm{HCN}$ and $\mathrm{SiO} / \mathrm{SiO}_{2}$ couples, in the gas excursions, $\mathrm{HS}$ and $\mathrm{H}_{2} \mathrm{~S}$ are coupled by the reaction

$\mathrm{HS}+\mathrm{H}_{2} \rightarrow \mathrm{H}_{2} \mathrm{~S}+\mathrm{H}$

which occurs with a $\approx 100 \%$ efficiency in both directions at any radius and for any $\mathrm{C} / \mathrm{O}$ ratio. The formation of HS must then take place in the immediate post-shock gas. Indeed, its formation after the shock front stems from the reaction

$\mathrm{H}+\mathrm{CS} \rightarrow \mathrm{HS}+\mathrm{C}$,

and to a less extent

$\mathrm{S}+\mathrm{CH} \rightarrow \mathrm{HS}+\mathrm{C}$,

whereas the destruction processes are the reverse reactions of the above channels. The destruction of HS involves atomic carbon, which has negligible abundances in O-rich and SE stars, thus allowing the survival of HS at large radii in these objects, especially because Reaction 26 keeps forming HS due to the high S content of the gas. For carbon stars, the atomic $\mathrm{C}$ attack tends to quickly destroy $\mathrm{HS}$ after its formation at $1 R_{\star}$.

Yamamura et al. (2000, Paper I) report the identification of ro-vibrational lines of HS in the IR spectrum of the S star R Andromedae. They derive an excitation temperature of $2200 \mathrm{~K}$ and a $\mathrm{HS} / \mathrm{H}$ abundance of $1 \times 10^{-7}$, and they found that the lines must come from a thin gas layer above the photosphere because of the lack of various velocity or temperature components in the HS lines. We see from Fig. 6 that the HS abundance with respect to $\mathrm{H}_{2}$ for a $\mathrm{C} / \mathrm{O}=0.98$ drops from $7 \times 10^{-6}$ at $1 R_{\star}$ to $1.3 \times 10^{-7}$ at $2.5 R_{\star}$ to reach $10^{-10}$ at $5 R_{\star}$. These abundances are in excellent agreement with the value derived by Yamamura et al., and we confirm the confinement of the HS distribution in the inner wind. As for $\mathrm{H}_{2} \mathrm{~S}$, we find that its distribution follows that of HS, and that the molecule is present in the inner wind of $\mathrm{M}$ and SE stars, but with lower abundances ranging from $\approx 10^{-7}$ to $10^{-9}$.

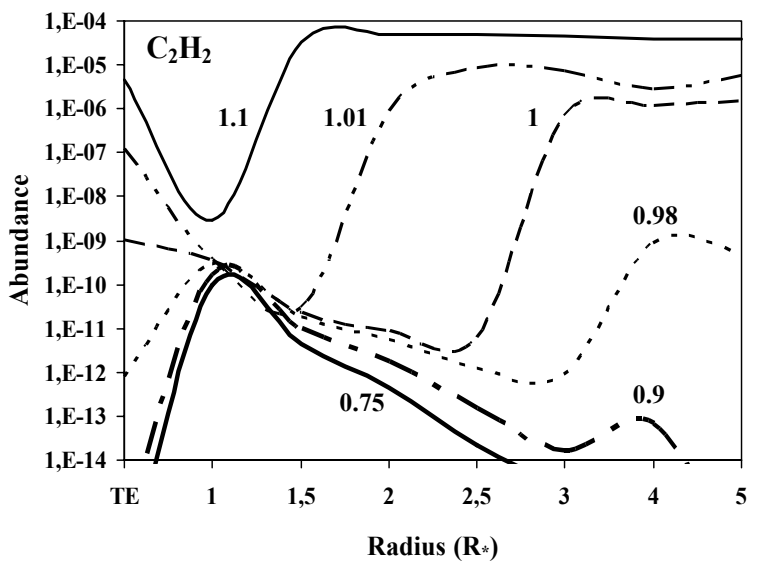

Fig. 7. $\mathrm{C}_{2} \mathrm{H}_{2}$ abundances versus $\mathrm{C} / \mathrm{O}$ ratio (curve labels) and stellar radius. TE abundances are also plotted on the left-hand side of the radius axis for comparison.

\subsection{Molecules typical of carbon chemistry}

We turn now to carbon-rich AGBs and present and discuss results for the most important molecules typical of a carbon-rich stellar environment.

\subsection{1. $\mathrm{C}_{2} \mathrm{H}_{2}$}

Acetylene $\mathrm{C}_{2} \mathrm{H}_{2}$ is an extremely important molecule in the wind of carbon-rich AGBs as it plays different roles depending on its position in the flow. Close to the star, it is conjectured that $\mathrm{C}_{2} \mathrm{H}_{2}$ triggers the formation of dust nucleation clusters such as large polycyclic aromatic hydrocarbons (PAHs) (Cherchneff et al. 1992; Cherchneff 1996; Cau 2002) and later contributes to the cluster growth via addition onto its surface. At larger radii, $\mathrm{C}_{2} \mathrm{H}_{2}$ is photo-dissociated to give rise to its radical $\mathrm{C}_{2} \mathrm{H}$ and a complex carbon chemistry leading to the formation of cyanopolyyne molecules and carbon chains (Cherchneff et al. 1993; Cherchneff \& Glassgold 1993; Millar et al. 2000). In this study, our model abundances for $\mathrm{C}_{2} \mathrm{H}_{2}$ are presented in Fig. 7 as a function of stellar radii and $\mathrm{C} / \mathrm{O}$ ratios. Very much like the $\mathrm{HCN} / \mathrm{CN}$ system studied in Sect. 4.2.3, acetylene formation occurs at any radii for all $\mathrm{C} / \mathrm{O}$ ratios via interchange reaction with its radical

$\mathrm{C}_{2} \mathrm{H}+\mathrm{H}_{2} \rightarrow \mathrm{C}_{2} \mathrm{H}_{2}+\mathrm{H}$

while the dominant destruction process is the opposite of the above reaction. However, $\mathrm{C}_{2} \mathrm{H}$ formation is linked to the small chain $\mathrm{C}_{2}$ just after the shock front by

$\mathrm{C}_{2}+\mathrm{H}_{2} \rightarrow \mathrm{C}_{2} \mathrm{H}+\mathrm{H}$

while $\mathrm{C}_{2}$ is formed simultaneously by the reverse of Reaction 10 . It is therefore this reaction which governs acetylene formation in AGBs, in part explaining the higher $\mathrm{C}_{2} \mathrm{H}_{2}$ abundances at $1 R_{\star}$ for $\mathrm{C} / \mathrm{O}=1.1$ observed in Fig. 7. Indeed, the higher atomic $\mathrm{C}$ content of the gas boosts the formation of $\mathrm{C}_{2}$ and $\mathrm{C}_{2} \mathrm{H}_{2}$. It is interesting to note, though, from Fig. 7, that the $\mathrm{C}_{2} \mathrm{H}_{2}$ abundance at $1 R_{\star}$ is the same for $\mathrm{M}$ and $\mathrm{S}$ stars, that is, for $\mathrm{C} / \mathrm{O}$ ratios varying from 0.75 to 1.01. While $\mathrm{C}_{2} \mathrm{H}_{2}$ abundances keep growing for $\mathrm{C} / \mathrm{O}=1.1$, we see that for other $\mathrm{C} / \mathrm{O}$ ratios, the growth occurs at larger radii, if any, and is preceded by a phase of destruction of the molecule. Indeed, once formed by Reaction $27, \mathrm{C}_{2} \mathrm{H}_{2}$ experienced the following additional destruction channel involving 


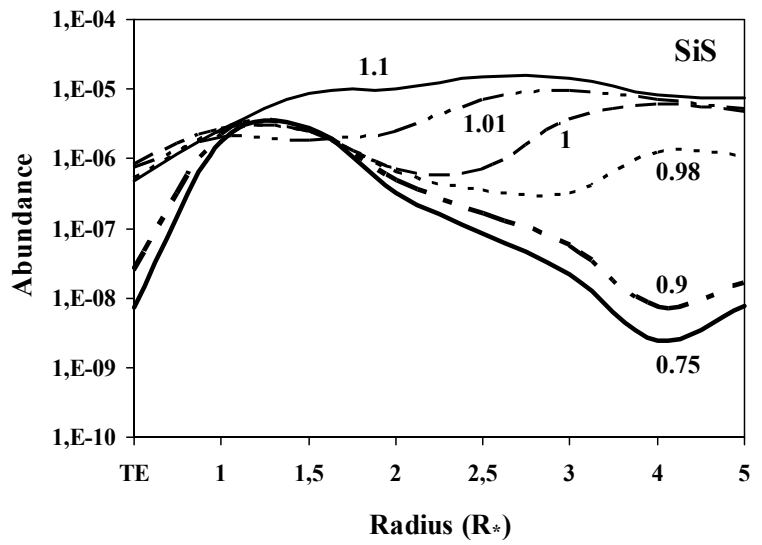

Fig. 8. SiS abundances versus $\mathrm{C} / \mathrm{O}$ ratio (curve labels) and stellar radius. TE abundances are also plotted on the left-hand side of the radius axis for comparison.

the formation of HS, and this reaction contributes up to $67 \%$ of acetylene destruction for $\mathrm{C} / \mathrm{O}=0.75$ :

$\mathrm{S}+\mathrm{C}_{2} \mathrm{H}_{2} \rightarrow \mathrm{C}_{2} \mathrm{H}+\mathrm{HS}$.

This destruction process would then explain why $\mathrm{C}_{2} \mathrm{H}_{2}$ formation is stopped in $\mathrm{O}$-rich at $1 R_{\star}$, while gradually progressing with increasing $\mathrm{C} / \mathrm{O}$ ratios, resulting in the absence of carbon dust in $\mathrm{M}$ stars. Furthermore, we see from Fig. 7 that for $\mathrm{S}$ stars of type $\mathrm{SC}(\mathrm{C} / \mathrm{O}=1.01), \mathrm{C}_{2} \mathrm{H}_{2}$ abundances increase rapidly around $1.5 R_{\star}$ to reach values of $\approx 1 \times 10^{-5}$, enabling a delayed carbon dust formation. To a smaller extent, the same conclusions can be drawn for $\mathrm{C} / \mathrm{O}=1$.

Absorption bands of acetylene around $14 \mu \mathrm{m}$ have been detected with the ISO SWS by Aoki et al. (1999) in several carbon stars and by Cernicharo et al. (1999) in IRC+ 10216. The latter derived a $\mathrm{C}_{2} \mathrm{H}_{2}$ abundance of $\approx 2.5 \times 10^{-5}$ in gas layers at temperatures $\approx 1700 \mathrm{~K}$ located between 1 and $3 R_{\star}$. The model acetylene abundance calculated by WC98 is 10 times larger, but this discrepancy could stem from the nucleation and condensation of dust, with possible deposition of $\mathrm{C}_{2} \mathrm{H}_{2}$ on the grain surfaces. There may also be additional destruction processes not considered in our neutral chemistry (see Sect. 4.5).

\subsubsection{SiS}

The chemistry of silicon sulphide SiS is linked to that of hydrogen sulfide HS and atomic $\mathrm{Si}$, and results are illustrated in Fig. 8. Its dominant formation process at small radii for all $\mathrm{C} / \mathrm{O}$ ratios involves the destruction of HS via

$\mathrm{Si}+\mathrm{HS} \rightarrow \mathrm{SiS}+\mathrm{H}$,

with an estimated reaction rate, based on the iso-valence of $\mathrm{C}$ and $\mathrm{Si}$, which is fast (see WC98), whereas the main destruction channel is the reverse reaction, which has an activation energy barrier, and is therefore efficient in hot dense gas layers only. At larger radii, the following reaction acts as an additional formation channel

$\mathrm{Si}+\mathrm{S}+\mathrm{M} \rightarrow \mathrm{SiS}+\mathrm{M}$

For C stars, the SiS formed close to the photosphere is carried away at large radii without suffering extra destruction reactions, resulting in a decreasing net formation rate and an almost constant $\mathrm{SiS}$ abundance, as illustrated in Fig. 8. For O-rich AGBs, on the other hand, an additional destruction reaction occurs at large radii, a situation similar to that of acetylene, via

$\mathrm{S}+\mathrm{SiS} \rightarrow \mathrm{S}_{2}+\mathrm{Si}$

resulting in a net destruction rate and a decrease in the SiS abundance.

SiS has long been observed at mm wavelengths in AGB stars. In particular, Bujarrabal et al. (1994) report its detection in some O-rich AGBs, including TX Cam and IK Tau, and in most of the carbon AGBs of their survey. They derived a higher SiS abundance in $\mathrm{C}$ stars (on average, $\approx 2 \times 10^{-6}$ ) than in $\mathrm{M}$ stars $\left(\approx 7 \times 10^{-7}\right)$, a trend confirmed by our present calculations. Their derived SiS abundances for IK Tau and TX Cam are always larger than those in our model, which predicts $\mathrm{SiS}$ as a parent molecule, but with inner abundance values injected at large radii much smaller than those observed in the outer stellar envelope. For TX Cam, our model abundance at $5 R_{\star}$ is $\approx 10^{-8}$, while that of Bujarrabal et al. is $10^{-6}$. However, our small inner abundances are not inconsistent with the picture that local chemical processes at large radii involving ion-molecule reactions must be efficient in building the SiS abundance derived from mm line observations.

Observations of carbon stars by Woods et al. (2003) report the detection of $\mathrm{SiS}$ in their star sample with abundances ranging from $3 \times 10^{-7}$ to $6 \times 10^{-6}$. The present model abundance calculated for our synthetic stellar model is $7 \times 10^{-6}$ at $5 R_{\star}$, therefore slightly higher than the observed values. This discrepancy can be due to either our estimated chemical reaction rates for Si chemistry, which could possibly be fine-tuned for better matches, or to a depletion effect of SiS due to freeze-out of the molecules on grains in the intermediate envelope. Both aspects should be further studied.

Finally, Aoki et al. (1998) report the detection of SiS first overtone bands in the $6.7 \mu \mathrm{m}$ region with the ISO SWS for the SC stars WZ Cas, but the bands could not be detected in the $\mathrm{N}$ stars of the sample. Our model predicts SiS values very similar for $\mathrm{SC}$ and $\mathrm{N}$ stars at small radii, confirming $\mathrm{SiS}$ as a parent molecule in these objects.

\subsection{Atomic species in the inner wind}

We see from above that in both O-rich and C-rich AGBs, some elements, namely $\mathrm{S}$ and $\mathrm{Si}$, play a crucial role in the formation or destruction of various key molecules, in particular $\mathrm{SiO}, \mathrm{CS}$, $\mathrm{SiS}$ and $\mathrm{C}_{2} \mathrm{H}_{2}$. Silicon abundances are illustrated in Fig. 9 as a function of $\mathrm{C} / \mathrm{O}$ ratio and position, and sulphur abundances are presented in Fig. 10. From our model, based on neutral chemistry, we find that $\mathrm{Si}$ and $\mathrm{S}$ have much lower abundances at $1 R_{\star}$ than given by TE calculations for any $\mathrm{C} / \mathrm{O}$ ratio. For carbon stars however, the $\mathrm{Si}$ abundance increases with radius to reach high values, whilst the abundance in M Miras keeps dropping.

For $\mathrm{C} / \mathrm{O}<1$, the dominant Si-bearing species is $\mathrm{SiO}$, and $\mathrm{Si}$ destruction stems from Reaction 5, while some Si returns to the gas via the opposite reaction. However, at larger radii, the dominant destruction process for $\mathrm{SiO}$ is not the reverse of Reaction 5 anymore, but Reaction 6 becomes responsible for $\mathrm{SiO}_{2}$ formation. Thus, $\mathrm{Si}$ is not returned to the gas, and its abundance drops to negligible values. For carbon stars, $\mathrm{SiO}_{2}$ formation is not favoured, and the reverse of Reaction 5 continues to return $\mathrm{Si}$ at large radii, resulting in higher $\mathrm{Si}$ abundances. The scenario for sulphur is quite different. For all $\mathrm{C} / \mathrm{O}$ ratios and close to the star, $\mathrm{S}$ atoms are consumed in the formation process of $\mathrm{SiS}, \mathrm{SO}$, and $\mathrm{HS}$, but some is returned to the gas via the respective reverse 


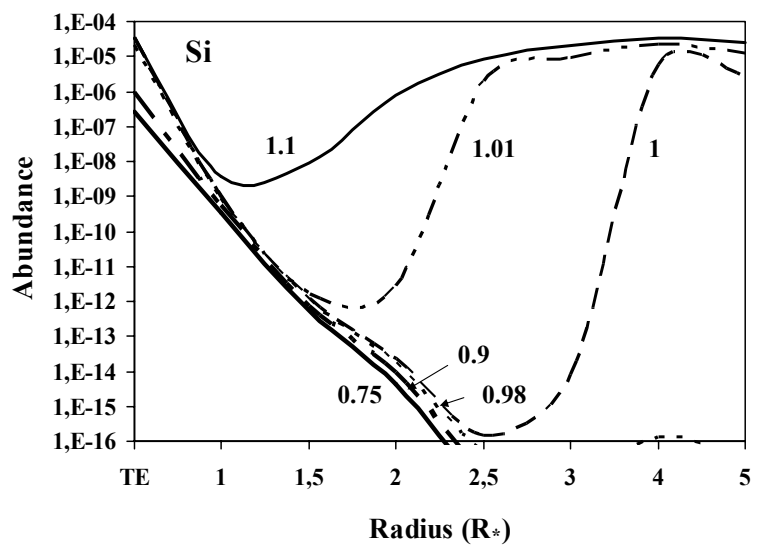

Fig. 9. $\mathrm{Si}$ abundances versus $\mathrm{C} / \mathrm{O}$ ratio (curve labels) and stellar radius. TE abundances are also plotted on the left-hand side of the radius axis for comparison.

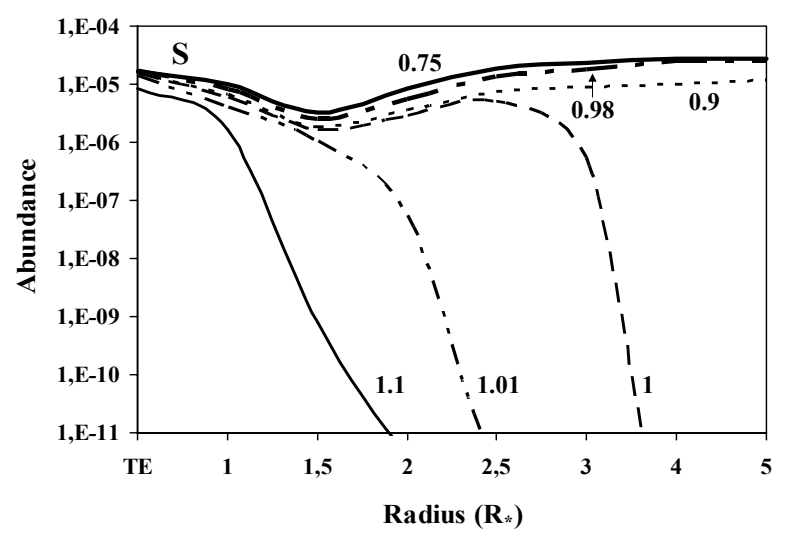

Fig. 10. $S$ abundances versus $C / O$ ratio (curve labels) and stellar radius. TE abundances are also plotted on the left-hand side of the radius axis for comparison.

chemical processes. At larger radii in carbon star, the formation of $\mathrm{SiS}$ is driven by Reaction 31, which stays efficient due to the return of $\mathrm{Si}$ atoms in the gas, as seen above. Therefore, $\mathrm{S}$ atoms get rapidly depleted in the gas, as illustrated in Fig. 10. In O-richAGBs on the other hand, the destruction of CS via Reaction 13 replenishes the gas of atomic $S$ to the level observed in Fig. 10.

Fine structure lines in emission of iron, sulphur, and silicon were detected in a sample of seven O-rich and C-rich AGBs by Aoki et al. (1998, Paper II) with the ISO SWS. They reported detection of emission lines in three objects only, the $\mathrm{M}$ star 30g Her and the carbon stars TX Psc and WZ Cas. In particular, the [SI] $25 \mu \mathrm{m}$ line was detected in TX Psc along with the [FeI] $24 \mu \mathrm{m}$ and the [FeII] $26 \mu \mathrm{m}$ lines, whereas the [SiII] $34.8 \mu \mathrm{m}$ line and two [FeII] at $26 \mu \mathrm{m}$ and $35.3 \mu \mathrm{m}$ were detected in $30 \mathrm{~g}$ Her. Neutral lines were thus observed in carbon stars when emission lines from ions were detected in $\mathbf{M}$ stars, reflecting the presence of a harsher environment in oxygen stars, possibly coming from chromospheric activity. Hass \& Glassgold (1993) have also observed the [SiII] fine structure lines in the Orich supergiant $\alpha$ Orionis whereas Castro-Carrizo et al. (2001) and Fong et al. (2001) report no detection of FIR atomic fine structure lines with the ISO LWS/SWS in oxygen and carbonrich AGBs.

At a first glance, these observations appear to contradict our model results. However, as mentioned before, we consider a neutral chemistry in our calculations, as we wish to understand the dominant chemical processes at play in molecular formation.
We should also include photo-ionization processes coming from chromospheric UV in M stars and photo-dissociation processes stemming from the soft UV radiation field of the star for carbon AGBs. Indeed, the detection in $30 \mathrm{~g}$ Her of [SiII] lines implies that $\mathrm{Si}$ must be present in the inner wind, a result in contradiction with that of our model. However, Si ions could come from the photo-dissociation of $\mathrm{SiO}$ ( $\mathrm{SiO}$ has a dissociation energy of $8 \mathrm{eV}$, close to the ionization potential of iron, whose ions are observed in $30 \mathrm{~g}$ Her) and the further ionization of Si atoms. As for carbon stars, neutral sulphur atoms could result from the photodissociation of SiS, as suggested by Oaki et al. (1998, Paper II).

An enhancement of atomic sulphur in the inner wind of carbon stars will result in the destruction of some of the acetylene formed close to the star, following Reaction 29, which would then reduce the gas $\mathrm{C}_{2} \mathrm{H}_{2}$ content. As seen above, the $\mathrm{C}_{2} \mathrm{H}_{2}$ abundance in IRC +10216 derived by Cernicharo et al. (1999) is 10 times smaller than the model value calculated by WC98, a result coherent with the existence of possible additional destruction channels for acetylene in the inner wind. Atomic sulphur would also react with molecular ions such as $\mathrm{CH}^{+}, \mathrm{HCN}^{+}$, and $\mathrm{OH}^{+}$to produce $\mathrm{HS}^{+}$and therefore enhance the abundances of $\mathrm{HS}$ and $\mathrm{HS}_{2}$ in the gas. On the other hand, we conjecture that the presence of $\mathrm{Si}$ ions in $\mathrm{M}$ stars would not much alter our neutral chemistry results, as $\mathrm{Si}^{+}$is expected to react with carbon chains, which are under-abundant in the inner wind, to form species of the silicon carbide family $\mathrm{SiC}_{x} / \mathrm{SiC}_{x} \mathrm{H}$. $\mathrm{Si}^{+}$will also transfer charge with other atomic species like $\mathrm{Na}$ or $\mathrm{Mg}$. A proper chemical model including chromospheric effects (三photo-ionization, photo-dissociation, and ion-neutral reactions), as well as the chemistry involving metals would confirm or disprove the above conjectures.

\subsection{The chemistry of $S$ stars}

Intrinsic $\mathrm{S}$ stars are believed to be a population of AGB stars for which the $\mathrm{C} / \mathrm{O}$ ratio is very close to unity, as a result of thermal pulsing and third dredge-up (Iben \& Renzini 1983). It is not clear yet if S stars represent objects in transition from M Miras to carbon stars or if they are a class of objects with a certain range of progenitor mass for which stellar evolution on the AGB stops at C/O close to unity (Bieging et al. 1994). Despite the fact that these stars appear to have mass loss rates comparable to other AGB stars, the nature of the dust they form is not clear because of the lack of net oxygen or carbon enhancement in their photosphere.

The dominant molecules formed in the inner wind of S stars in our calculations are listed in Table 5 as a function of $\mathrm{C} / \mathrm{O}$ ratio and position in the inner wind. For our SE star, that is $\mathrm{C} / \mathrm{O}=$ 0.98 , the molecular and atomic content of the inner envelope is close to that of O-rich Miras. For SC stars, i.e., $\mathrm{C} / \mathrm{O}=1.01$, the inner wind chemical composition is very similar to that of $\mathrm{C}$-rich AGBs. However, for $\mathrm{C} / \mathrm{O}=1$, we see that the inner wind has a chemical composition typical of both O-rich and C-rich AGB stars, with the presence of two shells, one close to the star with an O-rich molecular content, the second at larger radii and rich of $\mathrm{C}_{2} \mathrm{H}_{2}, \mathrm{SiS}$, and $\mathrm{Si}$ atoms, species typical of carbon stars. Therefore, the molecular content of S stars seems to be highly sensitive to the $\mathrm{C} / \mathrm{O}$ ratio and its vicinity to unity.

Although a $\mathrm{C} / \mathrm{O}$ ratio of 1 is unlikely to be represented by observable $\mathrm{S}$ stars, the above trends are interesting in terms of dust formation scenarios in AGB stars. In O-rich stars, we saw above that $\mathrm{SiO}$ forms constantly in the inner gas layers with high abundances, along with $\mathrm{SiO}_{2}$, which is present in small abundances of $\approx 1 \times 10^{-12}$. Although the dust nucleation and 
Table 5. Species present in the inner wind of $\mathrm{S}$ stars as a function of $\mathrm{C} / \mathrm{O}$ ratios and position.

\begin{tabular}{ccc}
\hline \hline $\mathrm{C} / \mathrm{O}$ & Species & Position from 1 to 5 $R_{\star}$ \\
\hline 0.98 & $\mathrm{CO}, \mathrm{SiO}, \mathrm{HCN}, \mathrm{CS}, \mathrm{H}_{2} \mathrm{O}, \mathrm{CO}_{2}, \mathrm{SO}, \mathrm{HS}, \mathrm{S}$ & any radius - similar to O-rich AGBs \\
\hline 1 & $\mathrm{CO}, \mathrm{SiO}, \mathrm{HCN}, \mathrm{CS}$ & any radius \\
& $\mathrm{CO}_{2}, \mathrm{SO}, \mathrm{H}_{2} \mathrm{O}, \mathrm{OH}, \mathrm{HS}, \mathrm{S}$ & present from 1 to $2,5 R_{\star}$ \\
& $\mathrm{C}_{2} \mathrm{H}_{2}, \mathrm{SiS}, \mathrm{Si}$ & present after $3 R_{\star}$ \\
\hline 1.01 & $\mathrm{CO}, \mathrm{SiO}, \mathrm{HCN}, \mathrm{CS}, \mathrm{C}_{2} \mathrm{H}_{2}, \mathrm{SiS}, \mathrm{Si}$ & any radius - similar to C-rich AGBs \\
\hline
\end{tabular}

condensation in O-rich AGBs has not yet been tackled in the inner, shocked regions, we know from the $9.7 \mu \mathrm{m}$ and $17.5 \mu \mathrm{m}$ features and the 33.6, 40.5, and $43 \mu \mathrm{m}$ emission bands that the nature of the dust formed is a mixture of amorphous silicate material (mainly Olivine $\left[\mathrm{MgFeSiO}_{4}\right]$ ) and crystalline silicates (forsterite $\left[\mathrm{Mg}_{2} \mathrm{SiO}_{4}\right]$, enstatite $\left[\mathrm{MgSiO}_{3}\right]$, diopside $\left[\mathrm{CaMgSi}_{2} \mathrm{O}_{6}\right]$, and water, Demyk et al. 2000). Under TE, the first condensate at high temperatures is corundum $\left(\mathrm{Al}_{2} \mathrm{O}_{3}\right)$ followed by various silicates. However, out of equilibrium, Donn \& Nuth (1985) reported that results of nucleation experiments on refractory materials were in disagreement with predictions of TE condensation schemes, a fact later confirmed by Rietmeijer et al. (1999). Furthermore, these experiments conducted with mixed $\mathrm{Mg}-\mathrm{SiO}$ and $\mathrm{Fe}-\mathrm{SiO}$ systems indicated that at high temperatures $(T>950 \mathrm{~K})$, pure $(\mathrm{SiO})_{x}$ clusters were more stable than mixed metal-SiO clusters. Although the chemical scheme for $(\mathrm{SiO})_{x}$ cluster formation in our present model is not considered, the high abundances of $\mathrm{SiO}$ formed in our gas and the trend of $\mathrm{SiO}$ conversion into $\mathrm{SiO}_{2}$ at $3 R_{\star}$ are coherent with the above picture.

For carbon-rich AGBs, we know that acetylene plays an important role in the dust nucleation and condensation stage, and the large $\mathrm{C}_{2} \mathrm{H}_{2}$ abundances found in this study comfirm this scenario.

For S stars however, the situation is far more complex. Skinner et al. (1990) report in their study of the IR spectrum of a large sample of $\mathrm{S}$ stars all possible dust configurations: some SE stars show clear silicate features, whilst other SE stars have the $\mathrm{SiC} 11.3 \mu \mathrm{m}$ feature in their spectrum along with the silicate bands, some SC stars show the SiC feature only and other $\mathrm{SC}$ stars have both $\mathrm{SiC}$ and silicate bands. We suggest from our study that most of these configurations could be explained by the gradually changing $\mathrm{C} / \mathrm{O}$ ratio of the star. For example, from inspection of Table 5, we see that SE stars showing the $\mathrm{SiC}$ feature could be stars with a $\mathrm{C} / \mathrm{O}$ ratio very close to but less than unity. The two-shell configuration mentioned above could lead to a first phase of carbon dust formation expelled in the flow prior to silicate formation.

As for SC stars with silicates bands, the explanation is less straightforward and different scenarios may play a role, including that of the presence of a companion star. Yamamura et al. (2000, Paper II) studied the near IR spectrum of V778 Cygni, a well-known silicate carbon star and showed that the ISO/SWS and IRAS/LRS spectra of the silicate emission bands were identical, implying no variation of the features over a 14 year period. They derived a silicate dust temperature of $\approx 600 \mathrm{~K}$ and some O-rich molecules were tentatively detected $\left(\mathrm{H}_{2} \mathrm{O}\right.$ and $\left.\mathrm{CO}_{2}\right)$. They ruled out the evolutionary scenario which predicts a possible fossil O-rich component coming from the previous Miras state of the star because the silicate bands would show some variation in this case due to a temperature change of the O-rich shell resulting from expansion. They further suggested the presence of an O-rich circumstellar disk around a companion star, located at $12 R_{\star}$ and constantly replenished with silicate material, to explain this steady behavior.
From our present study, we follow the suggestions of Skinner et al. (1990) and conjecture that carbon stars with silicate features could be SC stars with $\mathrm{C} / \mathrm{O}$ very close to unity for which the inner envelope shows a mixture of both O-rich and C-rich molecules. It is important to recall that the envelope region studied in this paper comprises gas layers which are gravitationally bound to the star in which the gas stays for a long period of time (several pulsation periods, that is, period $\geq 14$ years) with very specific ranges of parameters favorable to dust nucleation and condensation. Based on temperature constraints, dust formation could occur at any radius between 3 and $10 R_{\star}$, depending on the physical parameters of the star (in particular, the effective temperature and the shock velocity). The temperature of the gas excursions at the onset of dust formation can drop as low as $600 \mathrm{~K}$ in the adiabatic cooling post-shock region. At the outer edge of our inner wind, the freshly formed dust would drive the wind via radiation pressure on grains. A constantly replenished shell of dust arises from this scenario, and its composition varies depending on the non-expanding inner wind molecular composition. Those regions will experience slow and gradual changes in chemical composition when the photospheric $\mathrm{C} / \mathrm{O}$ ratio changes from 0.98 to 1.01 over a period typical of that of thermal pulses, i.e., $10 \times 10^{4}-10^{5}$ years. As seen from Table 5, the chemical composition of the inner wind can be dual for a long period of time compared to dust condensation time scales, with a mixture of O-rich and C-rich dust precursors. It is then reasonable to suggest that a two component (silicate-carbon) dust may form when the $\mathrm{C} / \mathrm{O}$ ratio approaches unity on the AGB. We by no means dispute the presence of disks in the environment of some stellar objects, but since there is no observational proof of a companion for V778 Cygni, more efforts should be dedicated to investigating the evolutionary scenario for this star with appropriate circumstellar and dust formation models.

\section{Summary and conclusions}

We have presented a chemical study of the quasi-static molecular layers, or extended atmosphere, or inner wind, of AGB stars, with a special emphasis on the interplay between stellar evolution and chemical composition. We summarize below the major findings:

1. Along the $\mathrm{AGB}$, the photospheric $\mathrm{C} / \mathrm{O}$ ratio is an indicator of the evolutionary stage of the star and describes the oxygen/carbon character of photospheric layers where thermal equilibrium holds. However, we find that the passage of shocks generated by stellar pulsation levels off the initial TE chemical discrepancy and forms a sub-layer just above the photosphere, with a very homogeneous chemical composition, independent of $\mathrm{C} / \mathrm{O}$ ratios. This homogeneity allows the formation of unexpected molecules (from a TE point of view), such as $\mathrm{HCN}, \mathrm{CS}$, and $\mathrm{CO}_{2}$, and implies that the dichotomy between a O-rich and a C-rich envelope appears later in the inner wind. In fact, in these layers, the chemical processes responsible for the formation of molecules are 
quite similar for the various $\mathrm{C} / \mathrm{O}$ ratios studied, leading to the formation of a few species ( $\mathrm{CO}, \mathrm{SiO}, \mathrm{HCN}$, and $\mathrm{CS}$ ) common to all AGB stars. In any case, molecular abundances derived from TE calculations should not be used in the interpretation of observational data which are not of the photosphere.

2. For all $\mathrm{C} / \mathrm{O}$ ratios, the radical $\mathrm{OH}$, and the silicon and sulphur atoms, present in the innermost part of our studied region, play a crucial role in the formation of important molecules. For $\mathrm{OH}$, they are $\mathrm{H}_{2} \mathrm{O}, \mathrm{CO} / \mathrm{CO}_{2}, \mathrm{SiO} / \mathrm{SiO}_{2}$, and $\mathrm{SO} / \mathrm{SO}_{2}$. In terms of dust precursor formation, the lack of $\mathrm{OH}$ in carbon stars inhibits the $\mathrm{SiO}$ trend of transforming into $\mathrm{SiO}_{2}$, resulting in a lack of silicate dust in these objects. Atomic Si forms $\mathrm{SiO}$ and $\mathrm{SiS}$. Finally, $\mathrm{S}$ atoms form $\mathrm{SO}$ and $\mathrm{CS}$, but destroy $\mathrm{CO}$ and $\mathrm{C}_{2} \mathrm{H}_{2}$. This destruction process is very efficient in stars for which $\mathrm{S}$ abundances are large and is then responsible for the inability of $\mathrm{M}$ stars to form carbon dust.

3. We find that certain molecules, in particular $\mathrm{CO}_{2}, \mathrm{SO}$, and HS, which are present only in M and SE stars, could be good tracers for discriminating between O-rich and C-rich AGBs. Moreover, HS has a very confined abundance distribution and is then an ideal candidate to probe the shock formation region. Observations of the fine structure emission lines of atoms and ions in the far infrared will be also extremely useful to better understand the nature of the chemical processes at play in the inner envelope.

4. The $\mathrm{S}$ stars show a chemical inner wind content similar to $\mathrm{M}$ or $\mathrm{C}$ stars except when their $\mathrm{C} / \mathrm{O}$ ratio is very close to unity, for which case they appear to have a dual O-rich/C-rich composition. This fact could help explaining the nature of SE stars with silicon carbide features, as well as silicate carbon stars.

Finally, we believe in the major importance of neutral-neutral processes in the inner wind of AGB stars. Although many reaction rates in our model are estimated because of a lack of laboratory data, we believe that our chemical model, along with our semi-analytical model of the shock dynamics, is robust enough as it can explain the formation of many observed molecules and reproduce abundances, that are, for the most part, in good agreement with values derived from available IR or mm observations. In the near future, high spectral resolution observations of rovibrational molecular transitions and atomic fine structure emission lines with HIFI/Herschel will shed light on the envelope's physical parameters and the chemical processes at play and will be extremely valuable for further theoretical modeling of the inner winds of AGBs. Time-resolved observations would also be particulary useful to probe these regions to understand the influence of shocks on the local chemistry. In parallel to observations, and to improve our study of the inner wind of AGB stars, we plan to incorporate the chemistry involving metals, the nucleation chemical steps to create small metal/silicate or metal carbide clusters, and the chemical and photo processes induced by the stellar radiation field and a possible chromospheric activity in our model.

Acknowledgements. The author wishes to thank A. G. G. M. Tielens for helpful comments on this study and A. Maeder for his careful reading of the manuscript.

\section{References}

Aoki, W., Tsuji, T., \& Ohnaka, K. 1998, A\&A, 333, L19 (Paper I) Aoki, W., Tsuji, T., \& Ohnaka, K. 1998, A\&A, 340, 222 (Paper II)
Aoki, W., Tsuji, T., \& Ohnaka, K. 1999, A\&A, 350, 945

Barlow, M. J., Nguyen-Q-Rieu, Truong-Bach, et al. 1996, A\&A, 315, L241 Beck, H. K. B., Gail, H.-P., Henkel, R., \& Sedlmayr, E. 1992, A\&A, 265, 626 Bertschinger, E., \& Chevalier, R. A. 1985, ApJ, 299, 167

Bieging, J. H., \& Latter, W. B. 1994, ApJ, 422, 765

Bieging, J. H., Knee, L. B. G., Latter, W. B., \& Olofsson, H. 1998, A\&A, 339, 811

Bieging, J. H., Shaked, S., \& Gensheimer, P. D. 2000, ApJ, 543, 897

Bowen, G. H. 1988, ApJ, 329, 299

Bujarrabal, V., Fuente, A., \& Omont, A. 1994, A\&A, 285, 247

Cami, J., Yamamura, I., de Jong, T., et al. 2000, A\&A, 360, 562

Castro-Carrizo, A., Bujarrabal, V., Fong, D., et al. 2001, A\&A, 367, 674

Cau, P. 2002, A\&A, 392, 203

Cernicharo, J., Yamamura, I., Conzález-Alfonso, E., et al. 1999, ApJ, 526, L41

Cernicharo, J. 1999, ASS, 263, 175

Cherchneff, I. 1995, ASS, 224, 379

Cherchneff, I. 1996, in Molecules in Astrophysics: Probes and Processes, ed. E. F. van Dischoeck (Dordrecht: Kluwer), Proc. IAU Symp., 178, 469 Cherchneff, I., \& Glassgold, A. E. 1993, ApJ, 419, L41

Cherchneff, I., Barker, J. R., \& Tielens, A. G. G. M. 1992, ApJ, 401, 269

Cherchneff, I., Glassgold, A. E., \& Mamon, G. 1993, ApJ, 410, 188

Demyk, K., Dartois, E., Wiesemeyer, H., Jones, A. P., \& d'Hendecourt, L. 2000, A\&A, 364, 170

Donn, B., \& Nuth, J. A. 1985, ApJ, 288, 187

Duari, D., \& Hatchell, J. 2000, A\&A, 358, L25

Duari, D., Cherchneff, I., \& Willacy, K. 1999, A\&A, 341, L47 (DCW99)

Conzález-Alfonso, E., \& Cernicharo, J. 1999, ApJ, 525, 845

Fleischer, A. J., Gauger, A., \& Sedlmayr, E. 1992, A\&A, 266, 321

Fong, D., Meixner, M., Castro-Carrizo, A., et al. 2001, A\&A, 367, 652

Fox, M. W., \& Wood, P. R. 1985, ApJ, 297, 455

González-Delgado, D., Olofsson, H., Kerschbaum, F., et al. 2003, A\&A, 411, 123

Hass, M. R., \& Glassgold, A. E. 1993, ApJ, 410, L111

Hinkle, K. H., Lebzelter, T., \& Scharlach, W. W. G. 1997, AJ, 114, 2686

Iben, I., \& Renzini, A. 1983, ARA\&A, 21, 271

Justtanont, K., de Jong, T., Helmich, F. P., et al. 1996, A\&A, 315, L217

Justtanont, K., de Jong, T., Tielens, A. G. G. M., Feuchtgruber, H., \& Waters, L. B. F. M. 2004, A\&A, 417, 625

Knapp, G. R., \& Morris, M. 1985, ApJ, 292, 640

Le Teuff, Y., Millar, T. J., \& Markwick, A. J. 2000, AAS, 146, 157

Lindqvist, M., Nyman, L.-A., Olofsson, H., \& Winnberg, A. 1988, A\&A, 205, L15

Markwick, A. J., \& Millar, T. J. 2000, A\&A, 359, 1162

Millar, T. J., Farquhar, P. R. A., \& Willacy, K. 1997, A\&AS, 121, 139

Millar, T. J., Herbst, E., \& Bettens, R. P. A. 2000, MNRAS, 316, 195

Millar, T., Flores, J. R., \& Markwick, A. J. 2001, MNRAS, 327, 1173

Nercessian, E., Omont, A., Benayoun, J. J., \& Guilloteau, S. 1989, A\&A, 210, 225

Neufeld, D. A., Chen, W., Melnick, G. J., et al. 1996, A\&A, 315, L237

Olofsson, H., Lindqvist, M., Nyman, L.-Å, Winnberg, A., \& Nguyen-Q-Rieu 1991, A\&A, 245, 611

Olofsson, H., Lindqvist, M., Nyman, L.-Å, \& Winnberg, A. 1998, A\&A, 329, 1074

Omont, A., Lucas, R., Morris, M., \& Guilloteau, S. 1993, A\&A, 267, 490

Perrin, G., Ridgway, S. T., Mennesson, B., et al. 2004, A\&A, 426, 279

Rietmeijer, F. J. M., Nuth, J. A., \& Karner, J. M. 1999, ApJ, 527, 395

Ryde, N., \& Eriksson, K. 2002, A\&A, 386, 874

Schöier, F. L., Olofsson, H., Wong, T., Lindqvist, M., \& Kerschbaum, F. 2004, A\&A, 422, 651

Skinner, C. J., Griffin, I., \& Whitmore, B. 1990, MNRAS, 243, 78

Tsuji, T. 2000, ApJ, 540, L99

Tsuji, T., Ohnaka, K., Aoki, W., \& Yamamua, I. 1997, A\&A, 320, L1

Truong-Bach, Sylvester, R. J., Barlow, M. J., et al. 1999, A\&A, 345, 925

Willacy, K., \& Millar, T. J. 1997, A\&A, 324, 237

Willacy, K., \& Cherchneff, I. 1998, A\&A, 330, 676 (WC98)

Woitke, P., Helling, C., Winters, J. M., \& Jeong, K. S. 1999, A\&A, 348, L17

Woods, P. M., Schöier, F. L., Nyman, L.-Å., \& Olofsson, H. 2003, A\&A, 402, 617

Yamamura, I., de Jong, T., Onaka, T., Cami, J., \& Waters, L. B. F. M. 1999, A\&A, 341, L9

Yamamura, I., Kawaguchi, K., \& Ridgway, S. T. 2000, ApJ, 528, L33 (Paper I) Yamamura, I., Dominik, C., de Jong, T., Waters, L. B. F. M., \& Molster, F. J. 2000, A\&A, 363, 629 (Paper II) 